OPEN ACCESS

Edited by:

Hugh Markus,

University of Cambridge,

United Kingdom

Reviewed by:

Maria Chalia,

University of Cambridge,

United Kingdom

Daniel Cozzolino,

Central Queensland University,

Australia

Felix Scholkmann,

University Hospital Zürich, Switzerland

Keum-Shik Hong,

Pusan National University, South

Korea

${ }^{*}$ Correspondence:

Pu Wang

hxkfwp@163.com

Specialty section

This article was submitted to

Stroke,

a section of the journal

Frontiers in Neurology

Received: 04 September 2018

Accepted: 16 January 2019

Published: 05 February 2019

Citation:

Yang $M$, Yang $Z$, Yuan $T$, Feng $W$ and Wang P (2019) A Systemic Review of

Functional Near-Infrared

Spectroscopy for Stroke: Current

Application and Future Directions.

Front. Neurol. 10:58.

doi: 10.3389/fneur.2019.00058

\section{A Systemic Review of Functional Near-Infrared Spectroscopy for Stroke: Current Application and Future Directions}

\author{
Muyue Yang ${ }^{1,2}$, Zhen Yang ${ }^{3}$, Tifei Yuan ${ }^{4}$, Wuwei Feng ${ }^{5}$ and Pu Wang ${ }^{1 *}$ \\ ${ }^{1}$ Department of Rehabilitation Medicine, Ruijin Hospital, Shanghai, China, ${ }^{2}$ School of Medicine, Shanghai Jiao Tong \\ University, Shanghai, China, ${ }^{3}$ Core Facility of West China Hospital, Sichuan University, Chengdu, China, ${ }^{4}$ Shanghai Mental \\ Health Centre, Shanghai Jiao Tong University School of Medicine, Shanghai, China, ${ }^{5}$ Department of Neurology, Medical \\ University of South Carolina, Charleston, SC, United States
}

Background: Survivors of stroke often experience significant disability and impaired quality of life. The recovery of motor or cognitive function requires long periods. Neuroimaging could measure changes in the brain and monitor recovery process in order to offer timely treatment and assess the effects of therapy. A non-invasive neuroimaging technique near-infrared spectroscopy (NIRS) with its ambulatory, portable, low-cost nature without fixation of subjects has attracted extensive attention.

Methods: We conducted a comprehensive literature review in order to review the use of NIRS in stroke or post-stroke patients in July 2018. NCBI Pubmed database, EMBASE database, Cochrane Library and ScienceDirect database were searched.

Results: Overall, we reviewed 66 papers. NIRS has a wide range of application, including in monitoring upper limb, lower limb recovery, motor learning, cortical function recovery, cerebral hemodynamic changes, cerebral oxygenation, as well as in therapeutic method, clinical researches, and evaluation of the risk for stroke.

Conclusions: This study provides a preliminary evidence of the application of NIRS in stroke patients as a monitoring, therapeutic, and research tool. Further studies could give more emphasize on the combination of NIRS with other techniques and its utility in the prevention of stroke.

\section{Keywords: near-infrared spectroscopy, stroke, motor recovery, cortical function, systemic review}

\section{INTRODUCTION}

Stroke, which refers to a medical condition in which insufficient brain blood supply results in cell death, is a major cause of death and disability worldwide $(1,2)$. Survivors are accompanied with the deterioration or loss of functions, for example, sensorimotor sequelae including motor weakness and impairment of voluntary motor control, spasticity, incoordination, apraxia, sensory loss/numbness, dysphagia, and dysarthria, and stroke could also lead to various cognitive and psychiatric deficits (3-5). These disfunctions are associated with cortical impairment due to insufficient blood supply and brain oxygenation. Therefore, monitoring the changes of brain circulation and oxygenation could timely reflect rehabilitation and recovery and the effect of therapy. 
Neuroimaging has been shown to be an effective monitoring and therapeutic tool, evaluating the evolution of neural activity and stroke rehabilitation and recovery (6). Traditional methods such as functional magnetic resonance imaging (fMRI), positron emission tomography, electroencephalography (EEG) and magnetoencephalography (MEG) - have provided considerable initial insight into brain changes during recovery. However, several shortcomings including a confining monitoring environment, subject head fixation and high cost have limited applications in tasks which require constant movement or realtime monitor $(7,8)$.

Near-infrared spectroscopy (NIRS), introduced in 1977 by Jöbsis et al. as a monitoring tool of cerebral and myocardial oxygenation (9), has partially overcome these difficulties. NIRS is a non-invasive neuroimaging tool that has several potential advantages including real-time monitor, low price, simplicity, portability, relatively small equipment, and it's almost completely safe and non-invasive nature (8).

NIRS can be divided into continuous wave NIRS (CW NIRS), time domain NIRS (TD NIRS), and frequency domain NIRS (FD NIRS). CW NIRS emits continuous wave and measures the changes in the intensity of the light that passed through the tissue, whereas TD NIRS utilizes a short pulse of laser light and measures the arrival times of photons emerging from the tissue. FD NIRS records the intensity of the detected light as well as the phase shift. These signals can then be converted to the concentration of oxygenated (oxy- $\mathrm{Hb}$ ) and deoxygenated hemoglobin (deoxy$\mathrm{Hb}$ ). One of the most common used algorithm is Modified Beer-Lambert Law (MBLL). CW NIRS could not measure absolute concentrations of oxy-Hb and deoxy- $\mathrm{Hb}$, because this method assumes a homogenous tissue which is not true. This does not change the results of qualitative analysis, but may lead to error in quantitative outcome. TD NIRS recording the temporal broadening of the pulse as it penetrates the investigated area allows accurate quantification of the concentrations and has better spatial resolution (10). Neural activity increases oxygen demands, thus increasing cerebral blood flow due to neurovascular coupling. NIRS could capture the changes of oxy- $\mathrm{Hb}$ and deoxy-Hb to infer changes in brain activity (6, 11). Recent years have witnessed rapid development of the techniques of NIRS from the single-location measurements to two dimensions and then three dimensions. One of its most promising application is in brain-computer interface (BCI) which was firstly introduced by Coyle et al. (12). BCI use brain activity to control external devices bypassing the peripheral nervous system. NIRS presents as a valuable tool in its brain signal acquisition for its non-invasive nature and real-time monitoring. However, fNIRS-BCI system is still mainly used in researches due to slow information transfer rate and low accuracy (13).

Stroke includes two major types: ischemic, due to lack of cerebral blood flow, and hemorrhagic, due to bleeding. During ischemic stroke cerebral blood supply is disrupted by narrowing of vessels caused by atherosclerosis or embolism. In hemorrhagic stroke caused by hypertension or ruptured aneurysm blood flow is reduced due to direct blood loss or vessel compression. In either case, a significant decline in blood supply could be observed. The reduction in cerebral oxygenation results in injury of neurovascular unit, decreased neuronal activity and accumulation of anaerobic metabolites. NIRS could monitor oxygenation signal changes, thus reflecting this pathophysiological process (1).

NIRS is well-established as a safe and effective monitoring tool for stroke recovery, including upper limb, lower limb recovery, motor learning, cortical function recovery (8), cerebral hemodynamic changes, cerebral oxygenation (14), therapy (15, 16), clinical researches and evaluation of the risk for stroke (17). Brain-computer interfaces (BCIs), a newly emerging tool, use brain activity to control external devices, facilitating paralyzed patients to interact with the environment. NIRS combined with BCI offers great potential as a therapeutic tool $(18,19)$. In addition, NIRS has used in several clinical studies $(20,21)$, reflecting hemodynamic or oxygenation changes of brain, as well as in evaluation of the risks for postoperative stroke and muscle metabolism (22). NIRS has shown to be an effective and promising method, however, its clinical value still remains controversial (23). Therefore, to address these discrepancies, we conducted this systematic review to summarize the existing application of NIRS in stroke patients.

\section{METHODS}

To evaluate how NIRS has been employed for surveillance and treatment of stroke patients, we conducted a systematic literature review of all published original research involving NIRS in stroke patients. The literature search, conducted in July 2018, began with an initial search of the NCBI Pubmed database using the following search terms: "('Spectroscopy, Near-Infrared' [MeSH Major Topic] OR "near-infrared spectroscopy"[Title/Abstract] OR fNIRS[Title/Abstract]) AND (stroke[MeSH Major Topic] OR “cerebrovascular disorders" [MeSH Major Topic] OR "brain infarction"[MeSH Major Topic])". Additional spot check searches stemming from key references in identified works were also carried out to further strengthen the literature review's reliability. Only English language articles published in peerreviewed journals were included. Then we searched EMBASE database, Cochrane Library and ScienceDirect database (Figure 1). All studies involving NIRS in stroke patients of any age were included. The details of included studies are presented in Tables 1-7. In total, our literature search identified 66 unique papers relating to stroke recovery, including motor recovery, motor learning, cortical function, hemodynamic changes, cerebral blood oxygen, Near-Infrared Spectroscopy-Based Brain-Computer Interface, and other applications in stroke patients, which were included for further analysis (Figure 2).

\section{NIRS FOR MOTOR RECOVERY}

We identified 14 papers (24-37) reporting on NIRS for motor recovery in a total of 399 patients, including upper limb function recovery, lower limb function recovery, balance control and motor learning (Table 1). In most patients, their motor function was significantly injured due to stroke, resulting in hemiparesis 


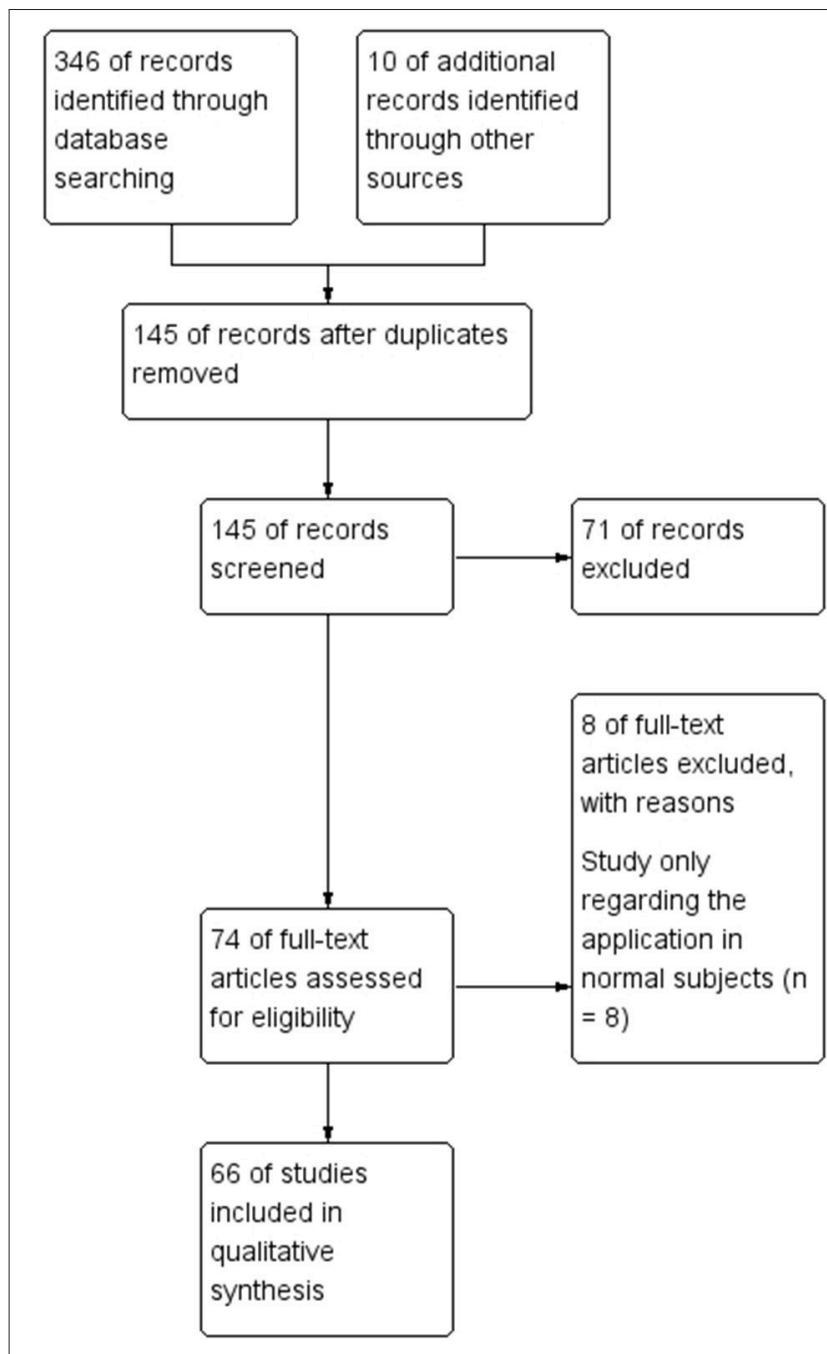

FIGURE 1 | Flowchart of literature search.

or tetraplegia. The concentration of oxy-Hb, deoxy-Hb, and total $\mathrm{Hb}$ were calculated by NIRS during various tasks, reflecting the activity of motor cortex. The probe is usually positioned at frontal regions, covering motor cortex, including bilateral premotor cortex (PMC), supplementary motor area (SMA), primary and secondary motor areas, and somatosensory areas.

Five papers (24-28) reported upper limb motor recovery (Table 1). In three of them, NIRS reflect cerebral cortical activity according to blood flow signals. Upper limb motor recovery has been shown to be associated with ipsilateral motor cortical compensation. Kato et al. evaluated the compensatory motor activation of cortical regions in 6 patients with cerebral infarction and 5 normal controls in comparison with functional MRI (fMRI) and demonstrated the contribution of ipsilateral motor cortical compensation or reorganization during recovery (24). Moreover, patients have shown longitudinal changes in functional laterality of activations in the primary sensorimotor cortex that at early stage affected hand movements activated bilateral sensorimotor cortices, whereas the activation pattern returned toward normal at later periods (25). Local perfusion may also play an essential role in motor recovery. Hara et al. studied the relationship between improvement in arm function and cerebral cortical perfusion changes during different tasks or treatments, in which electromyography-controlled functional electrical stimulation (EMG-FES) significantly increased cerebral blood flow, and may improve upper limb function (26). In another paper, the probe was placed on the flexor muscles of the forearm to detect changes in forearm tissue perfusion a single session of robot-assisted passive hand mobilization in 23 patients with subacute or chronic stroke patients. The results showed decreased upper limb heaviness and stiffness due to improved local circulation, therefore providing evidence that hand robotic assistance may help in the management of spasticity, heaviness, stiffness, and pain in patients with post-stroke hemiparesis (27). In addition, Mirror therapy (MT) has been shown that it could significantly improve upper limb motor function despite individual variance (28).

We found six papers (29-34) describing gait control and lower limb function recovery (Table 1). Miyai et al. demonstrated the contribution of motor cortices including PMC and pre-SMA in restoration of gait in patients with severe stroke (29). They then illuminated more detailed mechanism of gait recovery (30). By measuring cortical activities during hemiparetic gait on the treadmill, this study discovered gait improvement of asymmetry in medial primary sensorimotor cortex (SMC) activation and enhanced PMC activation in the affected hemisphere may play a role in locomotor recovery. However, the activation between stroke patients and normal subjects may be diverse. Another study further investigated the difference of cortical activation between patients and controls. The results revealed that during the acceleration phase both controls and patients showed obvious cortical activation in the medial SMC, SMA and prefrontal cortex (PFC). However, in the steady phase, brain activation attenuated in controls, but persisted in patients (31). Lin et al. compared cortical activation patterns during active cycling with and without speed feedback and during power-assisted cycling in stroke patients. Speed feedback may enhance the PMC activation and improve cycling performance in stroke patients (32). The reliability and feasibility of NIRS in monitoring lower limb movement recovery has been proved (33). Holtzer et al. conducted a research to confirm the posture first hypothesis that under dual-task walking conditions older adults prioritize gait over cognitive task performance. They assessed oxy- $\mathrm{Hb}$ levels in the PFC during normal walk and walk while talk (WWT) conditions in a large cohort of nondemented older adults, including normal subjects, central and peripheral neurological gait abnormalities (34). Interestingly, higher oxygenation levels were associated with better cognitive performance among normals, whereas, faster gait velocity in peripheral neurological gait abnormalities (NGA). This result suggested that presence of NGA may influence distribution of brain activities between gait and cognitive performance (34).

Balance problem is one of the major sequelae of stroke. Two papers $(35,36)$ focused on the mechanism of postural balance with external perturbation (Table 1). Mihara et al. Revealed broad cortical area, including the prefrontal, premotor, 
TABLE 1 | Summary of studies of NIRS in motor recovery.

\begin{tabular}{|c|c|c|c|c|c|c|c|}
\hline Study & $\begin{array}{c}\text { \# of } \\
\text { patients }\end{array}$ & Patients character & Etiology & Target area & Measurement & Method & Clinical applications \\
\hline \multicolumn{8}{|c|}{ UPPER LIMB RECOVERY } \\
\hline $\begin{array}{l}\text { Kato et al. } \\
(24)\end{array}$ & 6 & $\begin{array}{l}4 \text { men,2 women; } \\
\text { 59-79 years old }\end{array}$ & $\begin{array}{l}\text { Cerebral infarction of } \\
\text { the middle cerebral } \\
\text { artery territory }\end{array}$ & MCA & $\begin{array}{l}\Delta[\mathrm{O} x y-\mathrm{Hb}] \text { and } \\
\Delta[\mathrm{Deoxy}-\mathrm{Hb}]\end{array}$ & $\begin{array}{l}\text { Multi-channel } \\
\text { NIRS }\end{array}$ & $\begin{array}{l}\text { Evaluate the compensatory } \\
\text { motor activation of cortical } \\
\text { region }\end{array}$ \\
\hline $\begin{array}{l}\text { Takeda } \\
\text { et al. (25) }\end{array}$ & 5 & $\begin{array}{l}\text { one man, four } \\
\text { women, 52-67 } \\
\text { years old }\end{array}$ & Ischemic stroke & Bilateral SM1 & $\begin{array}{l}\Delta[\mathrm{Oxy}-\mathrm{Hb}] \text { and } \\
\Delta[\mathrm{Deoxy}-\mathrm{Hb}]\end{array}$ & 44-channel NIRS & $\begin{array}{l}\text { Monitor cerebral activation } \\
\text { and investigate the } \\
\text { longitudinal change }\end{array}$ \\
\hline $\begin{array}{l}\text { Hara et al. } \\
\text { et al. (26) }\end{array}$ & 16 & 14 men, 2 women & $\begin{array}{l}\text { Chronic stroke } \\
\text { patients with } \\
\text { residual hemiparesis }\end{array}$ & SMC & $\begin{array}{l}\Delta[\mathrm{Oxy}-\mathrm{Hb}] \text { and } \\
\Delta[\mathrm{Deoxy}-\mathrm{Hb}]\end{array}$ & 24-channel NIRS & $\begin{array}{l}\text { Investigate relationship } \\
\text { between hemiparetic arm } \\
\text { function improvement and } \\
\text { brain cortical perfusion } \\
\text { change during different } \\
\text { tasks }\end{array}$ \\
\hline $\begin{array}{l}\text { Gobbo } \\
\text { et al. ( } 27)\end{array}$ & 23 & $\begin{array}{l}13 \text { males; } 10 \\
\text { females, aged } 60.4 \\
\pm 13.2 \text { ) years } \\
\text { (range: } 40-84 \text { years }\end{array}$ & $\begin{array}{l}\text { Subacute or chronic } \\
\text { stroke }\end{array}$ & $\begin{array}{l}\text { Flexor muscles of the } \\
\text { forearm }\end{array}$ & $\begin{array}{l}\Delta[\mathrm{Oxy}-\mathrm{Hb}] \text { and } \\
\Delta[\mathrm{Deoxy}-\mathrm{Hb}]\end{array}$ & fNIRS & $\begin{array}{l}\text { Evaluate the acute effects } \\
\text { induced by a single session } \\
\text { of robot-assisted passive } \\
\text { hand mobilization on local } \\
\text { perfusion and upper limb } \\
\text { function }\end{array}$ \\
\hline $\begin{array}{l}\text { Brunetti } \\
\text { et al. (28) }\end{array}$ & 11 & $\begin{array}{l}\text { Six female, seven } \\
\text { male }\end{array}$ & $\begin{array}{l}\text { Subacute stroke } \\
\text { with severe upper } \\
\text { limb paresis }\end{array}$ & $\begin{array}{l}\text { Primary motor cortex } \\
\text { and precuneus }\end{array}$ & $\begin{array}{l}\Delta[\mathrm{Oxy}-\mathrm{Hb}] \text { and } \\
\Delta[\mathrm{Deoxy}-\mathrm{Hb}]\end{array}$ & fNIRS & $\begin{array}{l}\text { Investigate determinants of } \\
\text { efficacy of mirror therapy }\end{array}$ \\
\hline \multicolumn{8}{|c|}{ LOWER LIMB RECOVERY } \\
\hline $\begin{array}{l}\text { Miyai et al. } \\
\text { (29) }\end{array}$ & 6 & $\begin{array}{l}\text { Four men, two } \\
\text { women; } 57 \text { years old }\end{array}$ & $\begin{array}{l}\text { Stroke with } \\
\text { hemiplegia }\end{array}$ & $\begin{array}{l}\text { Bilateral frontoparietal } \\
\text { cortices covering } \\
\text { SMCs, PMCs, SMAs, } \\
\text { pre-SMAs, part of } \\
\text { prefrontal cortices, and } \\
\text { the superior parietal } \\
\text { lobes }\end{array}$ & $\begin{array}{l}\Delta[\mathrm{Oxy}-\mathrm{Hb}] \text { and } \\
\Delta[\mathrm{Deoxy}-\mathrm{Hb}]\end{array}$ & $\begin{array}{l}\text { Multi-channel } \\
\text { NIRS }\end{array}$ & $\begin{array}{l}\text { Investigate motor cortices } \\
\text { activation }\end{array}$ \\
\hline $\begin{array}{l}\text { Miyai et al. } \\
\text { (30) }\end{array}$ & 8 & $\begin{array}{l}5 \text { men, } 3 \text { women; } 4 \\
\text { with right and } 4 \text { with } \\
\text { left hemiparesis; } \\
\text { aged } 57 \text { years; }\end{array}$ & Stroke & $\begin{array}{l}\text { Bilateral frontoparietal } \\
\text { cortices covering the } \\
\text { primary SMC, PMC, } \\
\text { SMA, pre-SMA, part of } \\
\text { PFC, and the superior } \\
\text { parietal lobes }\end{array}$ & $\begin{array}{l}\text { Oxy-Hb, deoxy-Hb, } \\
\text { and total-Hb levels }\end{array}$ & $\begin{array}{l}\text { Multi-channel } \\
\text { NIRS }\end{array}$ & $\begin{array}{l}\text { Illuminate the mechanism of } \\
\text { gait recovery }\end{array}$ \\
\hline $\begin{array}{l}\text { Mihara } \\
\text { et al. (31) }\end{array}$ & 12 & $\begin{array}{l}11 \text { men,1 } \\
\text { woman;age range: } \\
\text { 12-74 years, mean } \\
\pm \text { S.D.: } 52.7 \pm 16.9 \\
\text { years }\end{array}$ & Infratentorial stroke & $\begin{array}{l}\text { Frontoparietal region } \\
\text { covering bilateral } \\
\text { PFC,the medial } \\
\text { PFC,SMA and medial } \\
\text { SMC }\end{array}$ & $\begin{array}{l}\Delta[\mathrm{Oxy}-\mathrm{Hb}] \text { and } \\
\Delta[\mathrm{Deoxy}-\mathrm{Hb}]\end{array}$ & 42-channel NIRS & $\begin{array}{l}\text { Investigate the difference of } \\
\text { cortical activation between } \\
\text { patients and controls }\end{array}$ \\
\hline $\begin{array}{l}\text { Lin et al. } \\
(32)\end{array}$ & 17 & $\begin{array}{l}16 \text { men, } 1 \text { woman; } \\
\text { age } 55.53 \pm 12.06\end{array}$ & $\begin{array}{l}\text { Subcortical stroke } \\
\text { with hemiparesis }\end{array}$ & $\begin{array}{l}\text { Frontoparietal region } \\
\text { covering SMC; bilateral } \\
\text { SMA and PMC }\end{array}$ & $\begin{array}{l}\Delta[\mathrm{Oxy}-\mathrm{Hb}] \text { and } \\
\Delta[\text { Deoxy-Hb]. }\end{array}$ & $\begin{array}{l}\text { Multichannel } \\
\text { frequency domain } \\
\text { NIRS }\end{array}$ & $\begin{array}{l}\text { Measure the cortical } \\
\text { activation patterns during } \\
\text { active cycling with and } \\
\text { without speed feedback }\end{array}$ \\
\hline $\begin{array}{l}\text { Rea et al. } \\
\text { (33) }\end{array}$ & 7 & 6 men, 1 woman & Chronic stroke & $\begin{array}{l}\text { Frontal regions, bilateral } \\
\text { PMC, SMA, primary } \\
\text { and secondary motor } \\
\text { areas, and } \\
\text { somatosensory areas }\end{array}$ & $\begin{array}{l}\Delta[\mathrm{Oxy}-\mathrm{Hb}] \text { and } \\
\Delta[\mathrm{Deoxy}-\mathrm{Hb}]\end{array}$ & fNIRS & $\begin{array}{l}\text { Assess the reliability and } \\
\text { feasibility of NIRS in clinical } \\
\text { usability in lower limb } \\
\text { movement preparation }\end{array}$ \\
\hline $\begin{array}{l}\text { Holtzer } \\
\text { et al. (34) }\end{array}$ & 236 & age $\geq 65$ years & $\begin{array}{l}167 \text { normal,40 } \\
\text { peripheral NGA,29 } \\
\text { central NGA }\end{array}$ & Forehead & $\begin{array}{l}\Delta[\mathrm{Oxy}-\mathrm{Hb}] \text { and } \\
\Delta[\mathrm{Deoxy}-\mathrm{Hb}]\end{array}$ & fNIRS & $\begin{array}{l}\text { Confirm the posture first } \\
\text { hypothesis }\end{array}$ \\
\hline \multicolumn{8}{|c|}{ BALANCE CONTROL } \\
\hline $\begin{array}{l}\text { Mihara } \\
\text { et al. (35) }\end{array}$ & 20 & $\begin{array}{l}15 \text { men and five } \\
\text { women; mean (SD) } \\
\text { age } 61.6(11.9) \\
\text { years }\end{array}$ & Stroke & Frontoparietal region & $\begin{array}{l}\Delta[\mathrm{Oxy}-\mathrm{Hb}] \text { and } \\
\Delta[\mathrm{Deoxy}-\mathrm{Hb}]\end{array}$ & fNIRS & $\begin{array}{l}\text { Evaluate cortical activation } \\
\text { associated with external } \\
\text { postural perturbation }\end{array}$ \\
\hline
\end{tabular}


TABLE 1 | Continued

\begin{tabular}{|c|c|c|c|c|c|c|c|}
\hline Study & $\begin{array}{c}\text { \# of } \\
\text { patients }\end{array}$ & Patients character & Etiology & Target area & Measurement & Method & Clinical applications \\
\hline $\begin{array}{l}\text { Fujimoto } \\
\text { et al. (36) }\end{array}$ & 20 & $\begin{array}{l}17 \text { men and } 3 \\
\text { women; mean }( \pm \\
\text { SD) age } 60.2( \pm 9.5) \\
\text { years }\end{array}$ & Subcortical stroke & Frontoparietal region & $\begin{array}{l}\Delta[\mathrm{Oxy}-\mathrm{Hb}] \text { and } \\
\Delta[\text { Deoxy-Hb]. }\end{array}$ & $\begin{array}{l}\text { Single-channel } \\
\text { NIRS }\end{array}$ & $\begin{array}{l}\text { Evaluate cortical activation } \\
\text { associated with external } \\
\text { postural perturbation }\end{array}$ \\
\hline \multicolumn{8}{|c|}{ MOTOR LEARNING } \\
\hline $\begin{array}{l}\text { Hatakenaka } \\
\text { et al. (37) }\end{array}$ & 12 & $\begin{array}{l}10 \text { men and } 2 \\
\text { women; mean }( \pm \\
\text { SD) age } 56( \pm 16) \\
\text { years }\end{array}$ & $\begin{array}{l}\text { Ataxia resulting from } \\
\text { infratentorial stroke }\end{array}$ & $\begin{array}{l}\text { Forehead, including } \\
\text { SMC, SMA, pre-SMA, } \\
\text { dorsal PMC, and } \\
\text { dorsolateral PFC }\end{array}$ & $\begin{array}{l}\Delta[\mathrm{Oxy}-\mathrm{Hb}] \text { and } \\
\Delta[\mathrm{Deoxy}-\mathrm{Hb}] .\end{array}$ & 42-channel NIRS & $\begin{array}{l}\text { Investigate impact of the } \\
\text { capacity for motor learning } \\
\text { on motor recovery }\end{array}$ \\
\hline
\end{tabular}

MCA, middle cerebral artery; SMC, sensorimotor cortex; PMC, premotor cortex; SMA, supplementary motor area; PFC, prefrontal cortex.

TABLE 2 | Summary of studies of NIRS in cortical function recovery.

\begin{tabular}{|c|c|c|c|c|c|c|c|}
\hline Study & $\begin{array}{c}\text { \# of } \\
\text { patients }\end{array}$ & Patients character & Etiology & Target area & Measurement & Method & Clinical applications \\
\hline $\begin{array}{l}\text { Park et al. } \\
\text { (38) }\end{array}$ & 1 & Male, 73 & Subcortical stroke & Bilateral motor area & $\begin{array}{l}\Delta[\mathrm{Oxy}-\mathrm{Hb}] \text { and } \\
\Delta[\mathrm{Deoxy}-\mathrm{Hb}]\end{array}$ & fNIRS & $\begin{array}{l}\text { Assess serial changes } \\
\text { during CIT }\end{array}$ \\
\hline $\begin{array}{l}\text { Schytz } \\
\text { et al. (39) }\end{array}$ & $\begin{array}{l}108 \text { (four } \\
\text { studies) }\end{array}$ & & Ischemic stroke & MCA & $\begin{array}{l}\Delta[\mathrm{Oxy}-\mathrm{Hb}] \text { and } \\
\Delta[\mathrm{Deoxy}-\mathrm{Hb}]\end{array}$ & $\begin{array}{l}\text { Multi-channel } \\
\text { NIRS }\end{array}$ & $\begin{array}{l}\text { Assess CA by recording } \\
\text { LFO }\end{array}$ \\
\hline $\begin{array}{l}\text { Han et al. } \\
(40)\end{array}$ & 10 & Age: $76.6 \pm 8.5$ year & Cerebral infarction & $\begin{array}{l}\text { Left and right prefrontal } \\
\text { lobes }\end{array}$ & $\mathrm{HbO} 2$ signals & fNIRS & $\begin{array}{l}\text { Assess the prefrontal } \\
\text { functional connectivity using } \\
\text { wavelet coherence analysis } \\
\text { of cerebral tissue } \\
\text { oxyhaemoglobin } \\
\text { concentration (Delta [HbO2]) } \\
\text { signals in elderly subjects } \\
\text { with cerebral infarction (Cl) } \\
\text { during the resting state }\end{array}$ \\
\hline $\begin{array}{l}\text { Cao et al. } \\
(41)\end{array}$ & 6 & $\begin{array}{l}3 \text { male, } 3 \text { female; } \\
\text { age } 10.2 \pm 2.1\end{array}$ & $\begin{array}{l}\text { Stroke with } \\
\text { hemiplegia }\end{array}$ & $\begin{array}{l}\text { Sensorimotor cortex, } \\
\text { covering PMC, SMA } \\
\text { and M1/S1 }\end{array}$ & $\begin{array}{l}\mathrm{Oxy}-\mathrm{Hb}, \text { deoxy-Hb, } \\
\text { and total-Hb levels }\end{array}$ & fNIRS & $\begin{array}{l}\text { Evaluate cortical plasticity } \\
\text { after CIT }\end{array}$ \\
\hline $\begin{array}{l}\text { Tan et al. } \\
(42)\end{array}$ & 12 & 6 men, 6 woman & Cerebral infarction & Bilateral forehead & $\begin{array}{l}\Delta[\mathrm{Oxy}-\mathrm{Hb}] \text { and } \\
\Delta[\mathrm{Deoxy}-\mathrm{Hb}]\end{array}$ & $\begin{array}{l}\text { Multi-channel } \\
\text { NIRS }\end{array}$ & $\begin{array}{l}\text { Evaluate functional } \\
\text { connectivity }\end{array}$ \\
\hline $\begin{array}{l}\text { Oldag } \\
\text { et al. (43) }\end{array}$ & 20 & $\begin{array}{l}12 \text { men, } 8 \text { women; } \\
\text { mean age } 55.3 \pm \\
12.5 \text { years }\end{array}$ & $\begin{array}{l}\text { Unilateral high-grade } \\
\text { steno-occlusion of } \\
\text { MCA }\end{array}$ & Bilateral MCA & $\begin{array}{l}\Delta[\mathrm{Oxy}-\mathrm{Hb}] \text { and } \\
\Delta[\mathrm{Deoxy}-\mathrm{Hb}]\end{array}$ & $\begin{array}{l}\text { Multi-channel } \\
\text { continuous wave } \\
\text { NIRS }\end{array}$ & Assess autoregulation delay \\
\hline $\begin{array}{l}\text { Moriya } \\
\text { et al. (44) }\end{array}$ & 11 & $\begin{array}{l}\text { Seven males, four } \\
\text { females, aged } 69.6 \\
\pm 12.0 \text { years }\end{array}$ & Stroke & Prefrontal cortex & $\begin{array}{l}\Delta[\mathrm{Oxy}-\mathrm{Hb}] \text { and } \\
\Delta[\mathrm{Deoxy}-\mathrm{Hb}]\end{array}$ & Two-channel NIRS & $\begin{array}{l}\text { Examine the acute effect of } \\
\text { physical exercise }\end{array}$ \\
\hline $\begin{array}{l}\text { Saita et al. } \\
\text { (45) }\end{array}$ & 2 & $\begin{array}{l}\text { 58-year-old male; } \\
\text { 74-year-old female }\end{array}$ & Cerebellar stroke & Bilateral frontal lobe & $\begin{array}{l}\Delta[\mathrm{Oxy}-\mathrm{Hb}] \text { and } \\
\Delta[\mathrm{Deoxy}-\mathrm{Hb}]\end{array}$ & fNIRS & Evaluate cortical activity \\
\hline $\begin{array}{l}\text { Mori et al. } \\
(46)\end{array}$ & 14 & $\begin{array}{l}12 \text { men and } 2 \\
\text { women; mean age, } \\
61.1 \pm 9.3 \text { years; } \\
\text { range, } 36-72 \text { years }\end{array}$ & Stroke & Prefrontal cortex & $\begin{array}{l}\Delta[\mathrm{O} x y-\mathrm{Hb}] \text { and } \\
\Delta[\mathrm{Deoxy}-\mathrm{Hb}]\end{array}$ & 16-channel NIRS & $\begin{array}{l}\text { Investigate the association } \\
\text { between PFC activity and } \\
\text { dual-task interference on } \\
\text { physical and cognitive } \\
\text { performance }\end{array}$ \\
\hline $\begin{array}{l}\text { White et al. } \\
(47)\end{array}$ & 2 & Infant & Perinatal stroke & Bilateraloccipital Lobe & Oxygenation & NIRS oximetry & $\begin{array}{l}\text { Investigate the functional } \\
\text { organization of the brain in } \\
\text { adults and infants }\end{array}$ \\
\hline
\end{tabular}

CIT, constraint-induced therapy; CA, cerebral autoregulation; LFO, low-frequency oscillations.

supplementary motor, and parietal cortical areas in both hemispheres, involved in balance control in poststroke patients (35). Among which, SMA was then demonstrated to be a crucial area-the increment of the postural-perturbation-related oxygenation signals in the SMA of the unaffected hemisphere was significantly correlated with the gain in balance function (36).

Motor learning is essential in function recovery, including motor adaptation, and motor sequence learning. Motor 
TABLE 3 | Summary of studies of NIRS in monitoring hemodynamic changes.

\begin{tabular}{|c|c|c|c|c|c|c|c|}
\hline Study & $\begin{array}{c}\text { \# of } \\
\text { patients }\end{array}$ & Patients character & Etiology & Target area & Measurement & Method & Clinical applications \\
\hline $\begin{array}{l}\text { Saitou } \\
\text { et al. (48) }\end{array}$ & 44 & & $\begin{array}{l}\text { Stroke with } \\
\text { hemiplegia }\end{array}$ & $\begin{array}{l}\text { Forehead on the injured } \\
\text { side }\end{array}$ & & & Detect cerebral perfusion \\
\hline $\begin{array}{l}\text { Terborg } \\
\text { et al. (49) }\end{array}$ & 13 & $\begin{array}{l}\text { Five female, eight } \\
\text { male; mean (SD)age, } \\
62.2 \text { (13.0) years }\end{array}$ & $\begin{array}{l}\text { Acute infarction in } \\
\text { the territory of MCA }\end{array}$ & $\begin{array}{l}\text { The territory of the } \\
\text { MCA }\end{array}$ & $\begin{array}{l}\text { Kinetics of an } \\
\text { intravenous bolus of } \\
\text { indocyanine green }\end{array}$ & NIRS & Detect cerebral ICG kinetics \\
\hline $\begin{array}{l}\text { Terborg } \\
\text { et al. (50) }\end{array}$ & 11 & $\begin{array}{l}6 \text { male, } 5 \text { female; } \\
\text { mean age, } 65.8 \pm \\
12.4 \text { years }\end{array}$ & $\begin{array}{l}\text { Acute infarction in } \\
\text { the territory of MCA }\end{array}$ & $\begin{array}{l}\text { The territory of the } \\
\text { MCA }\end{array}$ & $\begin{array}{l}\text { Kinetics of an } \\
\text { intravenous bolus of } \\
\text { indocyanine green }\end{array}$ & NIRS & $\begin{array}{l}\text { Measure effects of } \\
\text { head-of-bed (HOB) }\end{array}$ \\
\hline $\begin{array}{l}\text { Durduran } \\
\text { et al. (51) }\end{array}$ & 17 & $\begin{array}{l}7 \text { male, } 10 \text { female; } \\
44-93 \text { years }(65 \pm \\
16)\end{array}$ & $\begin{array}{l}\text { Acute ischemic } \\
\text { stroke }\end{array}$ & Frontal lobe & $\begin{array}{l}\Delta[\mathrm{Oxy}-\mathrm{Hb}] \text { and } \\
\Delta[\mathrm{Deoxy}-\mathrm{Hb}]\end{array}$ & Two-channel NIRS & Monitor blood flow signals \\
\hline $\begin{array}{l}\text { Muehlschlegel } \\
\text { et al. (52) }\end{array}$ & 12 & $\begin{array}{l}8 \text { male, } 4 \text { female; } \\
49-89 \text { years }\end{array}$ & Hemispheric strokes & Injured area & $\begin{array}{l}\text { Interhemispheric } \\
\text { correlation coefficient } \\
\text { (IHCC) }\end{array}$ & CW-NIRS & $\begin{array}{l}\text { Assess the cerebral } \\
\text { oxygenation oscillations }\end{array}$ \\
\hline Li et al. (53) & 10 & $\begin{array}{l}7 \text { male,3 female; age } \\
65 \pm 7\end{array}$ & $\mathrm{Cl}$ & Frontal lobe & $\begin{array}{l}\text { Cerebral oxygenation } \\
\text { oscillations }\end{array}$ & NIRS oximetry & $\begin{array}{l}\text { Monitor neuronal and } \\
\text { vascular signals }\end{array}$ \\
\hline $\begin{array}{l}\text { Leistner } \\
\text { et al. (54) }\end{array}$ & 6 & $\begin{array}{l}3 \text { male,3 female; age } \\
52 \pm 5\end{array}$ & $\begin{array}{l}\text { Subacute ischemic } \\
\text { stroke }\end{array}$ & Frontal lobe & $\begin{array}{l}\Delta[\mathrm{Oxy}-\mathrm{Hb}] \text { and } \\
\Delta[\mathrm{Deoxy}-\mathrm{Hb}]\end{array}$ & fNIRS & Monitor blood flow signals \\
\hline $\begin{array}{l}\text { Keller et al. } \\
\text { (55) }\end{array}$ & 1 & - & $\begin{array}{l}\text { Subarachnoid } \\
\text { hemorrhage }\end{array}$ & Injured area & $\begin{array}{l}\text { Kinetics of an } \\
\text { intravenous bolus of } \\
\text { indocyanine green }\end{array}$ & NIRS & Detect cerebral perfusion \\
\hline $\begin{array}{l}\text { Oldag } \\
\text { et al. (56) }\end{array}$ & 17 & $59.9 \pm 13.8$ & $\begin{array}{l}\text { Severe unilateral } \\
\text { stenosis or } \\
\text { segmental occlusion } \\
\text { of the MCA }\end{array}$ & $\begin{array}{l}\text { The cortical territory of } \\
\text { the MCA and its border } \\
\text { zone toward the } \\
\text { anterior cerebral artery }\end{array}$ & $\begin{array}{l}\Delta[\mathrm{Oxy}-\mathrm{Hb}] \text { and } \\
\Delta[\mathrm{Deoxy}-\mathrm{Hb}]\end{array}$ & $\begin{array}{l}\text { Multichannel } \\
\text { continuous wave } \\
\text { NIRS }\end{array}$ & $\begin{array}{l}\text { Assess the phase } \\
\text { relationship of prefrontal } \\
\text { tissue oxyhemoglobin } \\
\text { oscillations }\end{array}$ \\
\hline $\begin{array}{l}\text { Han et al. } \\
(57)\end{array}$ & 21 & $74.4 \pm 9.0$ years & $\mathrm{Cl}$ & Frontal lobe & $\begin{array}{l}\Delta[\mathrm{Oxy}-\mathrm{Hb}] \text { and } \\
\Delta[\mathrm{Deoxy}-\mathrm{Hb}]\end{array}$ & fNIRS & $\begin{array}{l}\text { Assess changes at } \\
\text { microvascular level }\end{array}$ \\
\hline $\begin{array}{l}\text { Zirak et al. } \\
\text { (58) }\end{array}$ & 1 & & Ischemic stroke & / & & & $\begin{array}{l}\text { Assess neurovascular } \\
\text { coupling }\end{array}$ \\
\hline $\begin{array}{l}\text { Dutta et al. } \\
\text { (59) }\end{array}$ & 4 & $\begin{array}{l}3 \text { males, } 1 \text { female } \\
\text { from age } 31 \text { to } 76\end{array}$ & $\begin{array}{l}\text { Chronic ischemic } \\
\text { stroke }\end{array}$ & Central site $\mathrm{Cz}$ & $\begin{array}{l}\text { Oxy- and deoxy- } \\
\text { hemoglobin }\end{array}$ & NIRS oximetry & $\begin{array}{l}\text { Investigate hemodynamic } \\
\text { and metabolic changes }\end{array}$ \\
\hline $\begin{array}{l}\text { Mitra et al. } \\
(60)\end{array}$ & 1 & Infant & Perinatal stroke & Either side of forehead & $\begin{array}{l}\text { Oxy- and deoxy- } \\
\text { hemoglobin }\end{array}$ & NIRS oximetry & \\
\hline $\begin{array}{l}\text { Cooper } \\
\text { et al. }(61)\end{array}$ & 4 & Infant & Perinatal stroke & Each temporal lobe & $\begin{array}{l}\text { Oxy- and } \\
\text { deoxy-hemoglobin }\end{array}$ & NIRS oximetry & $\begin{array}{l}\text { Investigate transient } \\
\text { hemodynamic events within } \\
\text { few hours in infants with } \\
\text { neurological damage }\end{array}$ \\
\hline
\end{tabular}

Cl, cerebral infarction; ICG, indocyanine green.

adaptation refers to the ability to compensate for environmental changes such as external force. Motor sequence learning is defined as learning of sequential motor actions, which is essential for activities of daily living. Impaired motor sequence learning may be correlated with reduced rehabilitation gains for ataxic patients with stroke (37).

\section{NIRS FOR CORTICAL FUNCTION RECOVERY}

The cortical functions including cortical plasticity, functional connectivity or cerebral autoregulation that could be reflected based on oxygenation levels are directly related to motor and cognitive recovery after stroke. We reviewed 10 papers (38-47) reporting cortical function recovery (Table 2).
Two studies have reported significant improvement in task performance or cortical plasticity after constraint-induced movement therapy $(38,41)$. In addition, fNIRS could assess the prefrontal functional connectivity using wavelet coherence analysis of cerebral tissue oxy-Hb concentration (40). A frequency-specific disruption in resting-state connectivity has been observed in cerebral infarction cases. fNIRS could also used in neonates as a bedside monitoring tool to early detect neurological deficiency and provide prognostic information (47). These evidence supported NIRS as a promising method for evaluating functional recovery (42).

Post-stroke patients exhibit changes in cerebral autoregulation (CA) which could be assessed by recording spontaneous oscillations in mean arterial blood pressure and cerebral blood flow. The etiology of oscillations remained unknown, however, its significance in reflecting CA has been demonstrated. NIRS 
TABLE 4 | Summary of studies of NIRS in monitoring cerebral blood oxygenation.

\begin{tabular}{|c|c|c|c|c|c|c|c|}
\hline Study & $\begin{array}{c}\text { \# of } \\
\text { patients }\end{array}$ & Patients character & Etiology & Target area & Measurement & Method & Clinical applications \\
\hline $\begin{array}{l}\text { Nemoto } \\
\text { et al. (62) }\end{array}$ & 2 & $\begin{array}{l}\text { 85-yr-old female; } \\
62-y r-o l d ~ f e m a l e\end{array}$ & $\begin{array}{l}\text { Infarction in the } \\
\text { territory of MCA }\end{array}$ & $\begin{array}{l}\text { Frontal-temporal or } \\
\text { frontal-parietal cortices }\end{array}$ & Oxygen saturation & NIRS oximetry & Monitor CBO changes \\
\hline $\begin{array}{l}\text { Damian } \\
\text { et al. (63) }\end{array}$ & 24 & $\begin{array}{l}15 \text { male, } 9 \text { female; } \\
43-77\end{array}$ & $\begin{array}{l}\text { Complete MCA } \\
\text { stroke and brain } \\
\text { swelling }\end{array}$ & Bilateral frontal lobe & Oxygen saturation & NIRS oximetry & Monitor CBO changes \\
\hline $\begin{array}{l}\text { Sakatani } \\
\text { et al. (64) }\end{array}$ & / & / & / & / & Oxygen saturation & NIRS oximetry & $\begin{array}{l}\text { Clarify CBO changes } \\
\text { occurring in stroke and } \\
\text { brain tumors }\end{array}$ \\
\hline $\begin{array}{l}\text { Aries et al. } \\
(65)\end{array}$ & 9 & $\begin{array}{l}3 \text { male, } 6 \text { female; } \\
\text { age } 71 \pm 10\end{array}$ & Acute stroke & Bilateral forehead & Oxygen saturation & NIRS oximetry & Monitor CBO changes \\
\hline $\begin{array}{l}\text { Mundiyanapure } \\
\text { et al. (66) }\end{array}$ & rath44 & 59-82 & $\begin{array}{l}\text { Acute ischemic } \\
\text { stroke }\end{array}$ & & Oxygen saturation & NIRS oximetry & Compare therapeutic effect \\
\hline $\begin{array}{l}\text { Liebig et al. } \\
(67)\end{array}$ & / & / & / & / & Cerebral oxygenation & NIRS oximetry & Compare therapeutic effect \\
\hline $\begin{array}{l}\text { Hametner } \\
\text { et al. (68) }\end{array}$ & 43 & $\begin{array}{l}22 \text { male, } 21 \text { female; } \\
65-79\end{array}$ & $\begin{array}{l}\text { Acute ischemic } \\
\text { stroke }\end{array}$ & Forehead & Cerebral oxygenation & NIRS oximetry & Measure rSO2 \\
\hline $\begin{array}{l}\text { Hashimoto } \\
\text { et al. (69) }\end{array}$ & 1 & 77-year-old man & $\mathrm{Cl}$ & Bilateral frontal lobe & $\begin{array}{l}\text { Regional cerebral } \\
\text { oxygen saturation } \\
\text { (rSO2) }\end{array}$ & NIRS oximetry & Monitor CBO changes \\
\hline $\begin{array}{l}\text { Ritzenthaler } \\
\text { et al. (70) }\end{array}$ & 3 & $\begin{array}{l}\text { 56-year-old man; } \\
\text { 52-year-old man; } \\
\text { 40-year-old } \\
\text { pregnant woman }\end{array}$ & MCA occlusion & Bilateral forehead & $\begin{array}{l}\text { Regional cerebral } \\
\text { oxygen saturation } \\
\text { (rSO3) }\end{array}$ & NIRS oximetry & Assess rSO2 changes \\
\hline $\begin{array}{l}\text { Nakamura } \\
\text { et al. }(71)\end{array}$ & 5 & $\begin{array}{l}\text { Four male, one } \\
\text { female, } 65.6 \pm 10.6 \\
\text { years }\end{array}$ & Stroke & Injured area & $\begin{array}{l}\Delta[\mathrm{Oxy}-\mathrm{Hb}] \text { and } \\
\Delta[\mathrm{Deoxy}-\mathrm{Hb}] .\end{array}$ & NIRS & $\begin{array}{l}\text { Evaluate effect of } \\
\text { revascularization on the } \\
\text { abnormal evoked CBO } \\
\text { responses }\end{array}$ \\
\hline $\begin{array}{l}\text { Vernieri } \\
\text { et al. (72) }\end{array}$ & 2 & $\begin{array}{l}\text { 58-year-old man; } \\
61 \text {-year-old man }\end{array}$ & Ischemic stroke & MCA & Cerebral oxygenation & NIRS oximetry & $\begin{array}{l}\text { Evaluate the effects of } \\
\text { hypercapnia }\end{array}$ \\
\hline $\begin{array}{l}\text { Moreau } \\
\text { et al. (73) }\end{array}$ & 5 & $\begin{array}{l}2 \text { male,3 female; } \\
\text { median age } 64 \\
\text { years old }\end{array}$ & Acute stroke & MCA & Cerebral oxygenation & NIRS oximetry & $\begin{array}{l}\text { Investigate brain } \\
\text { oxygenation in the first few } \\
\text { hours of stroke onset }\end{array}$ \\
\hline
\end{tabular}

$\mathrm{CBO}$, cerebral blood oxygenation.

TABLE 5 | Summary of studies of NIRS as therapeutic tool.

\begin{tabular}{|c|c|c|c|c|}
\hline Study & \# of patients & Etiology & Target area & Clinical applications \\
\hline Blokland et al. (15) & 10 & Stroke with tetraplegia & Motor cortex & $\begin{array}{l}\text { Test the feasibility of a combined } \\
\text { EEG-fNIRS system }\end{array}$ \\
\hline Mihara et al. (16) & 20 & Subcortical stroke & Frontoparietal region & $\begin{array}{l}\text { Investigate the effect of neurofeedback on } \\
\text { motor imagery }\end{array}$ \\
\hline
\end{tabular}

with a high time resolution is suggested as a promising tool for measuring oscillations, identifying risk and evaluating treatment strategies in patients with carotid artery disease and ischemic stroke $(39,43)$.

Mori et al. investigated dual-task interference on physical and cognitive performance in stroke patients. In consistent with previous studies, during dual-task walking, PFC activation might prioritize physical demands in stroke patients, but might prioritize cognitive demands in healthy subjects (46). Another study examined the acute effect of physical exercise on prefrontal cortex activity in post-stroke patients and demonstrated the therapeutic effect of moderate-intensity aerobic exercise by improving working memory performance (44).

\section{NIRS FOR HEMODYNAMIC CHANGES}

Fourteen papers (48-61) focusing on the applications of NIRS in monitoring hemodynamic changes in 158 stroke patients (Table 3). The probe usually positioned on the injured area of the brain, NIRS can detect cerebral perfusion timely in patients with acute infarction $(49,50)$ or steno-occlusive disease $(56)$ at bedside 
TABLE 6 | Summary of studies of NIRS in evaluating the risk for perioperative stroke.

\begin{tabular}{lcl}
\hline Study & \# of patients & Operation \\
\hline Olsson et al. (74) & 46 & Selective antegrade cerebral perfusion \\
Nakamura et al. (75) & 1 & CEA \\
Kellermann et al. (76) & $/$ & Cardiac surgery \\
Pennekamp et al. (77) & $/$ & Carotid endarterectomy \\
Ono et al. (78) & 234 & Cardiopulmonary bypass \\
Rummel et al. (79) & 35 & Balloon occlusion
\end{tabular}

CEA, Carotid Endarterectomy.

TABLE 7 | Summary of studies of other applications.

\begin{tabular}{|c|c|c|c|}
\hline References & $\begin{array}{l}\text { \# of } \\
\text { patients }\end{array}$ & Etiology & Clinical applications \\
\hline \multicolumn{4}{|c|}{ THE RISK FOR STROKE } \\
\hline Viola et al. (80) & 10 & Migraine & $\begin{array}{l}\text { Detect cerebral } \\
\text { microcirculation }\end{array}$ \\
\hline Li et al. (81) & 12 & Atherosclerosis & $\begin{array}{l}\text { Evaluate risk for } \\
\text { atherosclerotic stroke }\end{array}$ \\
\hline Pizza et al. (82) & 11 & $\begin{array}{l}\text { Acute/subacute middle } \\
\text { cerebral artery stroke }\end{array}$ & $\begin{array}{l}\text { Monitor oxygenation } \\
\text { level }\end{array}$ \\
\hline Liebig et al. (67) & 25 & Ischemic stroke & $\begin{array}{l}\text { Monitor signs of } \\
\text { ischemia during } \\
\text { neurovascular } \\
\text { procedures }\end{array}$ \\
\hline \multicolumn{4}{|l|}{ RESEARCH TOOL } \\
\hline Bönöczk et al. (20) & 43 & $\begin{array}{l}\text { Middle cerebral artery } \\
\text { stroke }\end{array}$ & $\begin{array}{l}\text { Measure cerebral blood } \\
\text { flow }\end{array}$ \\
\hline De et al. (21) & 3 & $\begin{array}{l}\text { Perinatal arterial } \\
\text { ischemic stroke }\end{array}$ & $\begin{array}{l}\text { Assess cerebral } \\
\text { oxygenation }\end{array}$ \\
\hline \multicolumn{4}{|l|}{ OTHERS } \\
\hline Selb et al. (83) & 36 & Stroke & $\begin{array}{l}\text { Investigate the effect of } \\
\text { MAs on "oscillation" } \\
\text { data }\end{array}$ \\
\hline Ferrante et al. (22) & 9 & Stroke & $\begin{array}{l}\text { Examine muscular } \\
\text { metabolism }\end{array}$ \\
\hline
\end{tabular}

MA: motion artifacts.

with indocyanine green (ICG) as tracer, providing a rapid, repeatable method without major side effects and transportation of critically ill patients $(48-50,56)$. Another study explored Diffuse correlation spectroscopy (DCS) and NIRS to measure effects of head-of-bed ( $\mathrm{HOB})$ positioning at different angles. The result showed increase in cerebral blood flow $(\mathrm{CBF})$ when the HOB is lowered from $30^{\circ}$ to $-5^{\circ}(51)$. Mitra et al. investigated metabolic and circulation changes following neonatal stroke in a term infant. Decreased oxy-Hb and clear asymmetry were noted (60).

NIRS could be used in intensive care unit as a neuromonitoring tool, reflecting blood flow signals $(52,55)$ as well as neuronal and vascular signals (54) in real-time. The interhemispheric correlation coefficient (IHCC) during physiological oscillations might be a new NIRS analysis tool

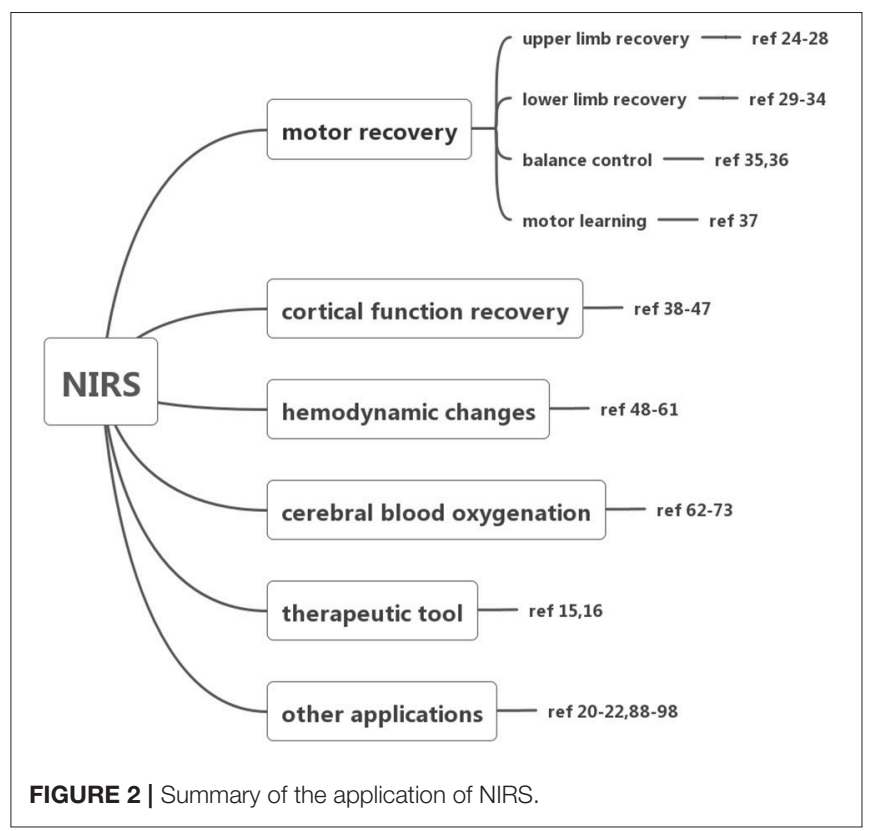

to quantify asymmetric microvascular hemodynamics (52). In addition, NIRS combined with diffuse correlation spectroscopy might provide hemodynamic changes at the microvascular level (58).

Spontaneous activity in prefrontal cerebral oxygenations has been demonstrated to be disturbed in the elderly and in patients with cerebral infarction (CI), which could be analyzed by continuous recording based on the wavelet transform of NIRS signals, could reflect resting state functional connectivity (53). Recent studies have shown the reduction of spontaneous oscillations in subjects with CI may suggest an increased stiffness in arterial vessels (53). Moreover, altered phase synchronization has been observed between the CI patients and healthy elderly indicates altered phase synchronization, indicating its potential utility to assessing atherosclerosis in high risk subjects for CI (57).

Hemodynamic changes recorded by NIRS could also provide critical information for other techniques. A method for EEGNIRS based assessment of neurovascular coupling (NVC) during anodal transcranial direct current stimulation (tDCS) was proposed in case series of four chronic ischemic stroke survivors (59). Cooper et al. used this EEG-NIRS system recorded transient haemodynamic events in neurologically compromised infants (61).

\section{NIRS FOR CEREBRAL BLOOD OXYGENATION}

We reviewed twelve (62-73) papers reporting on cerebral blood oxygenation (CBO) (Table 4). NIRS allows non-invasive measurement of regional cerebral oxygen saturation with high time resolution, reflecting cerebral perfusion and metabolism.

Oximetry by NIRS evaluates oxygenation changes in stroke patients (73) and the balance between regional oxygen supply 
and demand and assess functional state of the brain. In infarcted non-metabolizing brain, oxygen saturation may be near normal due to sequestered cerebral venous blood in capillaries and decreased consumption. In regionally or globally ischemic, but metabolizing brain, saturation decreases because oxygen supply is insufficient to meet metabolic demand (62). Several studies demonstrated good responsiveness of NIRS signal to oxygenation changes in stroke and brain tumors (63-65). Unlike fMRI which provides information mainly about concentration changes of deoxy-Hb that is paramagnetic,. NIRS detect both oxy-Hb and deoxy-Hb changes, thus providing more information (64). In addition, bilateral NIRS seems to be more useful on cerebral oxygenation than unilateral measurements and may help in prediction of the worsening of brain swelling (63).

One of the most important predictors of clinical outcome after acute ischemic stroke is recanalization. Endovascular treatment is one of major therapies of stroke patients. NIRS may be useful to monitor oxygenation during management of acute ischemic stroke (70). Nakamura et al. demonstrated that revascularization could improve the abnormal evoked-CBO response in ischemic stroke patients (71). Studies were designed to optimize clinical treatment with NIRS as a monitoring tool in measuring cerebral oximetry. However, whether patients suffering from acute ischemic stroke and undergoing endovascular recanalization should be treated under general anesthesia (GA) or conscious sedation (CS) remained a matter of debate. A prospective study with forty-four patients investigated their effects. The result showed CS groups required less vasopressor medication and had a higher mean blood pressure (66). Mechanical thrombectomy might have higher recanalization rates than intravenous or intraarterial thrombolysis, resulting in a better clinical outcome (67). Treatment of endovascular stroke often impedes neurologic assessment in patients who need sedation or general anesthesia. NIRS may fulfill this gap by measuring regional oxygen saturation (rSO2). Bi-channel rSO2-NIRS has potential for guiding neuroanesthesia and predicting outcome (68).

Cerebral hyperperfusion syndrome (CHS) was described in a case report of a 77-year-old man with acute cerebral infarction, which is known to be a rare but devastating complication of carotid artery revascularization. The patient underwent successful recanalization with thrombectomy by using a stent retriever for the middle cerebral artery and stent placement for the origin of the internal carotid artery. However, $12 \mathrm{~h}$ after treatment acute intracranial hemorrhage happened, which was considered to have been caused by CHS under the evaluation of NIRS. This case emphasized the significance of performing routine monitoring of regional cerebral oxygen saturation by NIRS to timely recognize CHS (69).

NIRS may be more useful in combination with other techniques. Vernieri et al. combined NIRS with transcranial Doppler to investigate the effects of hypercapnia on healthy individuals and patients of ischemic stroke (76). The results revealed that in healthy hemisphere scalp vessels dilated in response to hypercapnia, thus increasing cerebral blood flow, whereas in ischemic side this changes was negligible. This hybrid method could provide more information, with transcranial
Doppler recording subcortical changes and NIRS-cortical arterioles and capillary modifications (72).

\section{NIRS AS A THERAPEUTIC TOOL}

Despite several efficient therapies, neurorehabilitation is an intricate process requiring time and numerous factors. Given this circumstance, BCI which use brain activity to control external devices may be the only solution for patients with severe function deficiency, failing to interact with the environment and sustain normal daily lives.

BCI can be divided into two types, assistive BCI and rehabilitative BCI system. The assistive BCI system aims to substitute lost functions, for example, to achieve highdimensional movement control of robotic devices. In contrast, the rehabilitative BCI system aims to facilitate the restoration of brain function by normalizing the neurophysiologic activity (84). The patient's brain signals obtained via invasive or non-invasive means are acquired via amplifiers, filtered, and decoded using an online classification algorithm to control external devices. Distinct techniques have been explored to control a BCI, such as EEG and more recently NIRS.

NIRS is a relatively new measurement modality that offers a portable, low-cost, sensitive option for BCI development. The idea of using NIRS as an optical BCI has been introduced by Coyle in 2004 (85). Since then a number of studies (84-90) further investigated its application in $\mathrm{BCI}$, either examining the resulting signals for motor imagery or classifying the NIRS signals directly. Despite significant advantages because of non-invasive, portable nature of NIRS, there are several seconds of delay between neural activation and NIRS signal changes. To overcome these disadvantages of NIRS-based BCI systems, Siamac et al. combined EEG and NIRS measurements in BCI (91). Recently this hybrid EEG-fNIRS brain computer interface has attracted extensive attention and shown enhanced accuracy (92-97). Most of these studies, however, are limited in healthy population. Blokland et al. demonstrated feasibility and accuracy of using a combined EEG-fNIRS sensorimotor rhythms-based brain switch in patients with tetraplegia. A combined EEG-fNIRS system might be especially beneficial for patients whose classification performance based on EEG only are low (15). The recent study of Khan et al. Has shown the possibility of this hybrid EEGfNIRS system in controlling a quadcopter with fNIRS decoding mental arithmetic, counting, rotation, and word formation, EEG decoding eyeblinks and movement (92). The combination of EEG and fNIRS increases the number of control commands, improve classification accuracy and reduce the signal detection time (93). Further improvement in its efficacy requires proper brain region identification and feature selection (94).

In addition, NIRS was used in neurofeedback. Neurofeedback is a type of biofeedback that measures brain waves to produce a signal that can be used as feedback to modulate brain function voluntarily by the patients themselves. Real-time feedback of neural activity to the subjects may modulate plastic reorganization without external stimulation of the brain. NIRS, as a neurofeedback tool detecting blood flow signals, could 
improve cortical plasticity and enhance the therapeutic effect of motor imagery. In a pilot study twenty hemiplegic patients with subcortical stroke were analyzed and the results suggested that NIRS-mediated neurofeedback may enhance the efficacy of mental practice with motor imagery and augment motor recovery (16).

\section{OTHER APPLICATIONS}

Apart from applications above, NIRS has been used in evaluation of the risk for stroke, clinical studies and monitoring muscle metabolic indexes (Tables 6, 7).

Continuously monitoring oxygenation levels by NIRS during operation could diagnose and predict the occurrence of perioperative stroke. In a study of 46 patients underwent selective antegrade cerebral perfusion of the right subclavian artery, decreased regional cerebral tissue oxygen saturation was observed. Monitoring of regional cerebral oxygenation by NIRS allows detection of clinically important cerebral desaturation, and could predict perioperative neurologic sequelae (74). Two studies evaluated perioperative stroke after carotid endarterectomy $(75,77)$. NIRS could monitor cerebral changes during the surgery, providing immediate feedback to the treating physician and allowing prompt corrections. Multichannel NIRS detects watershed-type posterior perfusion defects, superior to single-channel (75). NIRS is also of great importance during cardiopulmonary bypass (78), cardiac surgery (76), and vessel occlusion $(67,79)$. Treatment of intracranial aneurysms by surgical clipping carries a risk of intraoperative ischemia, caused mainly by prolonged temporary occlusion of cerebral arteries. NIRS monitoring of cerebral blood flow (CBF) during surgery may decrease the risk (67). Another study proposed multichannel CW NIRS during endovascular neuroradiologic interventions requiring temporary balloon occlusion of arteries supplying the cerebral circulation (79). Based on these studies, NIRS plays an essential role in monitoring perioperative stroke, however, a combination of several monitoring modalities may enhance accuracy and require further investigations.

Studies have also evaluated the risk for stroke in other diseases. We reviewed three papers. Viola et al. assessed hemodynamic changes in migraine patients. The results showed a mild vasoconstriction, which may be the possible explanation of the association between migraine and an increased risk for ischemic stroke (80). The spontaneous cerebral oscillations based on the wavelet transform of NIRS signals were assessed to evaluate risk for atherosclerotic stroke (81). Sleep-disordered breathing (SDB) could lead to cerebral deoxygenation, resulting in stroke outcome. By monitoring oxygenated state of the brain, NIRS could recognize these conditions at early stage (82).

In addition, NIRS presents as an effective research tool in clinical studies. The concentration of oxy- $\mathrm{Hb}$ and deoxy-Hb obtained by NIRS could be measured, calculated and analyzed, evaluating hemodynamic, oxygenation changes and cortical function, thus widely used in assessing the effects of different therapies or the evolution of diseases $(20,21)$. Ferrante et al. combined functional electrical stimulation and time-domain
NIRS (TD NIRS) to examine muscular metabolism on poststroke patients and the result indicated metabolic dysfunction of muscles seemed to be local and unilateral (22).

\section{DISCUSSION AND FUTURE DIRECTIONS}

NIRS, a non-invasive neuroimaging technique with the advantages of low price, simplicity, portability and small devices, has a wide range of utility (48). The existing clinical literature suggests NIRS plays an important role in monitoring motor recovery, including upper limb (24-28), lower limb recovery (29-34), balance control $(35,36)$, motor learning (37), cortical function recovery (38-47), cerebral hemodynamic changes (48$61)$, cerebral oxygenation $(62-73)$, therapy $(15,16)$, and other applications $(20-22,67,74-83)$. In particular, its ambulatory real-time measurement without the fixation of position provides great value in tasks requiring constant movement, for example, gait control.

NIRS is applied in healthy population at first and then used in stroke subjects. By monitoring of NIRS could reflect the function recovery and the therapeutic effect in patients; compared with normal people, it can be used to study the pathophysiological mechanism of disease and predict the risk, serving as an effective monitoring and research tool. In addition, it can be used as therapeutic tool combined with BCI and neurofeedback, but the current studies mainly limited in healthy people, however, the results have shown potential value, requiring further investigations in stroke patients.

Despite its non-invasive, portable and ambulatory nature, its accuracy is influenced by several factors, thus limiting its further application. fNIRS detects neurovascular coupling and its accuracy may be impaired by extracerebral and systemic changes such as blood pressure and concentration of CO2 $(98,99)$. This problem confounds all hemodynamic-based neuroimaging methods, like fMRI. However, due to its high sensitivity, the slight blood fluctuations in the scalp may cause "false positives and negatives." We generally assumed the signal changes are only related to neural activity. However, in reality, the fNIRS signals are composed of six components: evoked/non-evoked cerebral neuronal changes and evoked/non-evoked cerebral/extracerebral systemic changes $(23,100)$. These shortages could be minimized by careful study design, improved fNIRS techniques and statistical processing (23). A modified fNIRS techniques with higher resolution is needed. The newly proposed fNIRS-based bundled-optode method used 32 optodes could increase spatial resolution and this method can be extended for 3D fNIRS imaging $(101,102)$. As to obtain higher temporal resolution for ANIRS-BCI, several studies demonstrated the detection of the initial dip may be useful. The initial dip refers to an early small decrease of the concentration of oxy-Hb and is closely associated with neuronal metabolism. fNIRS-based BCI using initial dip detection could reduce the command generation time and improve temporal resolution $(103,104)$. In addition, longitudinal studies, for example, measuring with NIRS/fNIRS on one subject every day for a week could provide deeper insight the dynamic changes of brain, and as time prolonged 
the influence of confounding factors could be minimized. Furthermore, stroke has specific circadian and circannual rhythms. Most of strokes occurred in the morning between 7 a.m. and noon (105). Therefore, the time of measurement may influence the efficacy of NIRS and determining the optimal time window for measurements might be one aspect for optimization of NIRS. Apart from "false positives and negatives," NIRS provides no anatomical information, thus must use scalp anatomy to locate the position where the signals arise $(52,106)$. At present, inhibitory and excitatory activity cannot be discriminated, which requiring the support of other techniques (107).

Considering the inevitable shortcomings, one promising prospect is the combination of different neuroimaging techniques, including diffusion-tensor imaging (DTI), magnetic resonance spectroscopy (MRS), ligand-based positron emission tomography (PET), single-photon emission computed tomography (SPECT), functional magnetic resonance imaging (fMRI), NIRS, and EEG. Conjugated with other techniques, the surveillance might be more comprehensive and accurate. Moreover, at present most studies have focused on its application in stroke recovery, its ability to assess the occurrence of stroke, for example, during operation and in people with higher risk, is of great significance. Further researches could emphasize NIRS in the prevention of stroke as well as stroke recovery and prognosis.

NIRS could also used in combination with neurostimulation tools. Medical communities have been interested in electrical stimulation for a long time. Recently transcranial direct current stimulation (tDCS), which was firstly used in humans in 2,000 by Nitsche et al. (108), has shown to be an appealing therapeutic tool in neurological disorders. It emits small amounts of electric current to modulate the excitability of neuron and has shown

\section{REFERENCES}

1. Donnan GA, Fisher M, Macleod M, Davis SM. Stroke. Lancet (2008) 371:1612-23. doi: 10.1016/S0140-6736(08)60694-7

2. Feigin V, Roth G, Naghavi M, Parmar P, Krishnamurthi R, Chugh $\mathrm{S}$, et al. Global burden of stroke and risk factors in 188 countries, during 1990-2013: a systematic analysis for the Global Burden of Disease Study 2013. Lancet Neurol. (2016) 15:913-24. doi: 10.1016/S1474-4422(16) 30073-4

3. Schweizer TA, Schweizer TA, Fachgebiete H. The behavioral consequences of stroke (2013).

4. Handley A, Medcalf P, Hellier K, Dutta D. Movement disorders after stroke. Age Ageing (2009) 38:260-66. doi: 10.1093/ageing/afp020

5. Elias G, Namasivayam AA, Lozano AM. Deep brain stimulation for stroke: current uses and future directions. Brain Stimulat. (2017) 11:3-28. doi: 10.1016/j.brs.2017.10.005

6. Cyrous A, O'Neal B, Freeman WD. New approaches to bedside monitoring in stroke. Exp Rev Neurotherapeut. (2012) 12:915-28. doi: 10.1586/ern. 12.85

7. Strangman G, Goldstein R, Rauch SL, Stein J. Near-infrared spectroscopy and imaging for investigating stroke rehabilitation: test-retest reliability and review of the literature. Arch Phys Med Rehabil. (2006) 87(12 Supp_S):S12-9. doi: 10.1016/j.apmr.2006.07.269 to be effective in many neurological disorders, including stroke, Parkinson's disease, schizophrenia, depression and addiction (109). Several studies has suggested its ability to change oscillatory neural activity in resting state, functional connectivity and event related desynchronization/synchronization which are often injured in patients with motor disorders (110). It could also influence membrane potentials of pyramidal neurons and induce long lasting effects on cortical plasticity by modifying $\mathrm{N}$ methyl-D-aspartate (NMDA) receptor and revealed changes in resting state oscillatory neural activity, functional connectivity, and event related activity during cognitive tasks $(111,112)$. These results could partially explain its therapeutic effects, but its exact mechanism still remain unclear. Neuroimaging tool, including fMRI, PET, EEG and NIRS could partially illuminate the neurophysiological changes, providing valuable insights into brain changes during electrical stimulation. However, the application of fMRI and PET are restricted due to bulky and immobile properties. EEG are portable and suitable in combination with tDCS, but its accuracy may be diminished by disturbance of artifacts. fNIRS, based on the measurements of light intensity, are not affected by electrically induced artifacts and might be most desirable tool in tDCS researches (113).

\section{AUTHOR CONTRIBUTIONS}

PW contributed to the conception of the study. MY contributed significantly to manuscript preparation and wrote the manuscript. ZY, TY, and WF revised the manuscript and approved the final version.

\section{FUNDING}

Gaofeng Medical Technology Talents Project (grant number: 2018061).

8. Mihara M, Miyai I. Review of functional near-infrared spectroscopy in neurorehabilitation. Neurophotonics (2016) 3:031414. doi: 10.1117/1.NPh.3.3.031414

9. Jöbsis FF. Noninvasive, infrared monitoring of cerebral and myocardial oxygen sufficiency and circulatory parameters. Science (1977) 198:1264. doi: 10.1126/science.929199

10. Boas DA, Elwell CE, Ferrari M, Taga G. Twenty years of functional nearinfrared spectroscopy: introduction for the special issue. Neuroimage (2014) 1:1-5. doi: 10.1016/j.neuroimage.2013.11.033

11. Scholkmann F, Kleiser S, Metz AJ, Zimmermann R, Mata Pavia J, Wolf U, et al. A review on continuous wave functional near-infrared spectroscopy and imaging instrumentation and methodology. Neuroimage (2014) 85(Pt 1):6-27. doi: 10.1016/j.neuroimage.2013.05.004

12. Coyle S, Ward T, Markham C, McDarby G. On the suitability of near-infrared (NIR) systems for next-generation brain-computer interfaces. Physiol Meas. (2004) 25:815-22.

13. Naseer N, Hong KS. fNIRS-based brain-computer interfaces: a review. Front Hum Neurosci. (2015) 9:3. doi: 10.3389/fnhum.2015.00003

14. Ludlow CL. Using Neuroimaging and neuromodulation to study changes in brain functioning with therapy[C]// seminars in speech and language. Semin Speech Lang (2012) 333:175-87. doi: 10.1055/s-0032-1320038

15. Blokland Y, Spyrou L, Thijssen D, Eijsvogels T, Colier W, Floor-Westerdijk $\mathrm{M}$, et al. Combined EEG-fNIRS decoding of motor attempt and imagery for 
brain switch control: an offline study in patients with tetraplegia. IEEE Trans Neural Syst Rehabil Eng. (2014) 22:222-9. doi: 10.1109/TNSRE.2013.2292995

16. Mihara M1, Hattori N, Hatakenaka M, Yagura H, Kawano T, Hino T, et al. Near-infrared spectroscopy-mediated neurofeedback enhances efficacy of motor imagery-based training in poststroke victims: a pilot study. Stroke (2013) 44:1091-8. doi: 10.1161/STROKEAHA.111.674507

17. Pennekamp CW, Bots ML, Kappelle LJ, Moll FL, de Borst GJ. The value of near-infrared spectroscopy measured cerebral oximetry during carotid endarterectomy in perioperative stroke prevention. A review. Eur J Vasc Endovasc Surg. (2009) 38:539. doi: 10.1016/j.ejvs.2009.07.008

18. Daly JJ, Wolpaw JR. Brain-computer interfaces in neurological rehabilitation. Lancet Neurol. (2008) 7:1032-43. doi: 10.1016/S1474-4422(08)70223-0

19. Dobkin BH. Brain-computer interface technology as a tool to augment plasticity and outcomes for neurological rehabilitation. J Physiol. (2007) 579:637-42. doi: 10.1113/jphysiol.2006.123067

20. Bönöczk P, Panczel G, Nagy Z. Vinpocetine increases cerebral blood flow and oxygenation in stroke patients: a near infrared spectroscopy and transcranial Doppler study. Eur J Ultrasound (2002) 15:85-91. doi: 10.1016/S0929-8266(02)00006-X

21. De Vis JB, Petersen ET, Kersbergen KJ, Alderliesten T, de Vries LS, van Bel F, et al. Evaluation of perinatal arterial ischemic stroke using noninvasive arterial spin labeling perfusion MRI. Pediat Res. (2013) 74:30713. doi: 10.1038/pr.2013.111

22. Ferrante S, Contini D, Spinelli L, Pedrocchi A, Torricelli A, Molteni F, et al. Monitoring muscle metabolic indexes by time-domain near-infrared spectroscopy during knee flex-extension induced by functional electrical stimulation. J Biomed Optics (2009) 6629:044011. doi: 10.1117/1.3183802

23. Tachtsidis I, Scholkmann F. False positives and false negatives in functional NIRS: issues, challenges and the way forward. Neurophotonics (2016) 3:030401. doi: 10.1117/1.NPh.3.3.030401

24. Kato H, Izumiyama M, Koizumi H, Takahashi A, Itoyama Y. Near-infrared spectroscopic topography as a tool to monitor motor reorganization after hemiparetic stroke: a comparison with functional MRI. Stroke (2002) 33:2032-6.

25. Takeda K1, Gomi Y, Imai I, Shimoda N, Hiwatari M, Kato H. Shift of motor activation areas during recovery from hemiparesis after cerebral infarction: a longitudinal study with near-infrared spectroscopy. Neurosci Res. (2007) 59:136-44. doi: 10.1016/j.neures.2007.06.1466

26. Hara Y, Obayashi S, Tsujiuchi K, Muraoka Y. The effects of electromyography-controlled functional electrical stimulation on upper extremity function and cortical perfusion in stroke patients. Clin Neurophysiol. (2013) 124:2008-15. doi: 10.1016/j.clinph.2013.03.030

27. Gobbo M, Gaffurini P, Vacchi L, Lazzarini S, Villafane J, Orizio C, et al. Hand passive mobilization performed with robotic assistance: acute effects on upper limb perfusion and spasticity in stroke survivors. BioMed Res Int. (2017) 2017:1-6. doi: 10.1155/2017/2796815

28. Brunetti M, Morkisch N, Fritzsch C, Mehnert J, Steinbrink J, Niedeggen $\mathrm{M}$, et al. Potential determinants of efficacy of mirror therapy in stroke patients - A pilot study. Restorat Neurol Neurosci. (2015) 33:421-34. doi: 10.3233/RNN-140421

29. Miyai I, Yagura H, Oda I, Konishi I, Eda H, Suzuki T, et al. Premotor cortex is involved in restoration of gait in stroke. Ann Neurol. (2010) 52:188-94. doi: 10.1002/ana.10274

30. Miyai I, Yagura H, Hatakenaka M, Oda I, Konishi I, Kubota K. Longitudinal optical imaging study for locomotor recovery after stroke. Stroke (2003) 34:2866. doi: 10.1161/01.STR.0000100166.81077.8A

31. Mihara M, Miyai I, Hatakenaka M, Kubota K, Sakoda S. Sustained prefrontal activation during ataxic gait: a compensatory mechanism for ataxic stroke?. Neuroimage (2007) 37:1338-45. doi: 10.1016/j.neuroimage.2007.06.014

32. Lin PY, Chen JJ, Lin SI. The cortical control of cycling exercise in stroke patients: an fNIRS study. Hum Brain Mapp. (2014) 34:2381-90. doi: $10.1002 / \mathrm{hbm} .22072$

33. Rea M, Rana M, Lugato N, Terekhin P, Gizzi L, Brötz D, et al. Lower limb movement preparation in chronic stroke: a pilot study toward an fNIRSBCI for gait rehabilitation. Neurorehabil Neural Repair (2014) 28:564-75. doi: $10.1177 / 1545968313520410$

34. Holtzer R, Verghese J, Allali G, Izzetoglu M, Wang C, Mahoney JR. Neurological gait abnormalities moderate the functional brain signature of the posture first hypothesis. Brain Topogr. (2015) 29:334-43. doi: 10.1007/s10548-015-0465-z

35. Mihara M, Miyai I, Hattori N, Hatakenaka M, Yagura H, Kawano T, et al. Cortical control of postural balance in patients with hemiplegic stroke. NeuroReport (2012) 23:314-9. doi: 10.1097/WNR.0b013e32835 $1757 \mathrm{~b}$

36. Fujimoto $H$, Mihara $M$, Hattori $N$, Hatakenaka $M$, Kawano $T$, Yagura $\mathrm{H}$, et al. Cortical changes underlying balance recovery in patients with hemiplegic stroke. Neuroimage (2014) 85:547-54. doi: 10.1016/j.neuroimage.2013.05.014

37. Hatakenaka M, Miyai I, Mihara M, Yagura H, Hattori N. Impaired motor learning by a pursuit rotor test reduces functional outcomes during rehabilitation of poststroke ataxia. Neurorehabil Neural Repair (2012) 26:293. doi: $10.1177 / 1545968311412053$

38. Park SW, Butler AJ, Cavalheiro V, Alberts JL, Wolf SL. Changes in serial optical topography and TMS during task performance after constraintinduced movement therapy in stroke: a case study. Neurorehabil Neural Repair (2004) 18:95-105. doi: 10.1177/0888439004265113

39. Schytz HW, Hansson A, Phillip D, Selb J, Boas DA, Iversen HK, et al. Spontaneous low-frequency oscillations in cerebral vessels: applications in carotid artery disease and ischemic stroke. J Stroke Cerebrovasc Dis. (2010) 19:465-74. doi: 10.1016/j.jstrokecerebrovasdis.2010.06.001

40. Han Q, Zhang M, Li W, Gao Y, Xin Q, Wang Y, et al. Wavelet coherence analysis of prefrontal tissue oxyhaemoglobin signals as measured using nearinfrared spectroscopy in elderly subjects with cerebral infarction. Microvasc Res. (2014) 95:108-15. doi: 10.1016/j.mvr.2014.08.001

41. Cao J, Khan B, Hervey N, Tian F, Delgado MR, Clegg NJ, et al. Evaluation of cortical plasticity in children with cerebral palsy undergoing constraintinduced movement therapy based on functional near-infrared spectroscopy. J Biomed Optics (2015) 20:046009. doi: 10.1117/1.JBO.20.4.046009

42. Tan Q, Zhang M, Wang Y, Zhang M, Wang Y, Xin Q, et al. Frequencyspecific functional connectivity revealed by wavelet-based coherence analysis in elderly subjects with cerebral infarction using NIRS method. Med Phys. (2015) 42:5391-403. doi: 10.1118/1.4928672

43. Oldag A, Neumann J, Goertler M, Hinrichs H, Heinze HJ, Kupsch A, et al. Near-infrared spectroscopy and transcranial sonography to evaluate cerebral autoregulation in middle cerebral artery steno-occlusive disease. J Neurol. (2016) 263:2296-301. doi: 10.1007/s00415-016-8262-5

44. Moriya M, Aoki C, Sakatani K. Effects of physical exercise on working memory and prefrontal cortex function in post-stroke patients. Adv Exp Med Biol. (2016) 923:203. doi: 10.1007/978-3-319-38810-6_27

45. Saita K, Ogata T, Watanabe J, Tsuboi Y, Takahara M, Inoue T, et al. Contralateral cerebral hypometabolism after cerebellar stroke: a functional near-infrared spectroscopy study. J Stroke Cerebrovasc Dis. (2017) 26:e69-71. doi: 10.1016/j.jstrokecerebrovasdis.2017

46. Mori T, Takeuchi N, Izumi SI. Prefrontal cortex activation during a dual task in patients with stroke. Gait Posture (2018) 59:193-8. doi: 10.1016/j.gaitpost.2017.09.032

47. White BR, Liao SM, Ferradal SL, Inder TE, Culver JP. Bedside optical imaging of occipital resting-state functional connectivity in neonates. Neuroimage (2012) 59:2529-38. doi: 10.1016/j.neuroimage.2011.08.094

48. Saitou H, Yanagi H, Hara S, Tsuchiya S, Tomura S. Cerebral blood volume and oxygenation among poststroke hemiplegic patients: effects of 13 rehabilitation tasks measured by near-infrared spectroscopy. Arch Phys Med Rehabil. (2000) 81:1348-56. doi: 10.1053/apmr.2000.9400

49. Terborg C, Bramer S, Harscher S, Simon M, Witte OW. Bedside assessment of cerebral perfusion reductions in patients with acute ischaemic stroke by near-infrared spectroscopy and indocyanine green. J Neurol Neurosurg Psychiat. (2004) 75:38-42.

50. Terborg C, Gröschel K, Petrovitch A, Ringer T, Schnaudigel S, Witte OW, et al. Noninvasive assessment of cerebral perfusion and oxygenation in acute ischemic stroke by near-infrared spectroscopy. Eur Neurol. (2009) 62:338-43. doi: 10.1159/000239794

51. Durduran T, Zhou C, Edlow BL, Yu G, Choe R, Kim MN. Transcranial optical monitoring of cerebrovascular hemodynamics in acute stroke patients. Optics Express (2009) 17:3884-902.

52. Muehlschlegel S, Selb J, Patel M, Diamond SG, Franceschini MA, Sorensen $A G$, et al. Feasibility of NIRS in the neurointensive care unit: a pilot study in 
stroke using physiological oscillations. Neurocritical Care (2009) 11:288-95. doi: 10.1007/s12028-009-9254-4

53. Li Z, Wang Y, Li Y, Wang Y, Li J, Zhang L. Wavelet analysis of cerebral oxygenation signal measured by near infrared spectroscopy in subjects with cerebral infarction. Microvasc Res. (2010) 80:142-7. doi: 10.1016/j.mvr.2010.02.004

54. Leistner S, Sander-Thoemmes T, Wabnitz H, Moeller M, Wachs M, Curio G, et al. Non-invasive simultaneous recording of neuronal and vascular signals in subacute ischemic stroke. Biomed Tech. (2011) 56:85-90. doi: $10.1515 / \mathrm{bmt} .2011 .002$

55. Keller E, Froehlich J, Muroi C, Sikorski C, Muser M. Neuromonitoring in intensive care: a new brain tissue probe for combined monitoring of intracranial pressure (ICP) cerebral blood flow (CBF) and oxygenation. Acta Neurochirurg Suppl. (2011) 110:217-20. doi: 10.1007/978-3-7091-0356-2_39

56. Oldag A, Goertler M, Bertz AK, Schreiber S, Stoppel C, Heinze HJ, et al. Assessment of cortical hemodynamics by multichannel near-infrared spectroscopy in steno-occlusive disease of the middle cerebral artery. Stroke (2012) 43:2980. doi: 10.1161/STROKEAHA.112.656710

57. Han Q, Li Z, Gao Y, Li W, Xin Q, Tan Q, et al. Phase synchronization analysis of prefrontal tissue oxyhemoglobin oscillations in elderly subjects with cerebral infarction. Med Phys. (2014) 41:102702. doi: 10.1118/1.4896113

58. Zirak P, Delgado-Mederos R, Dinia L, Carrera D, Martí-Fàbregas J, Durduran T. Transcranial diffuse optical monitoring of microvascular cerebral hemodynamics after thrombolysis in ischemic stroke. J Biomed. Optics (2014) 19:18002. doi: 10.1117/1.JBO.19.1.018002

59. Dutta A, Jacob A, Chowdhury SR, Das A, Nitsche MA. EEG-NIRS based assessment of neurovascular coupling during anodal transcranial direct current stimulation - a stroke case series. J Med Syst. (2015) 39:36. doi: 10.1007/s10916-015-0205-7

60. Mitra S, Bale G, Meek J, Mathieson S, Uria C, Kendall G, et al. In vivo measurement of cerebral mitochondrial metabolism using broadband near infrared spectroscopy following neonatal stroke. Adv Exp Med Biol. (2016) 876:493. doi: 10.1007/978-1-4939-3023-4_62

61. Cooper RJ, Hebden JC, O’Reilly H, Mitra S, Michell AW, Everdell NL, et al. Transient haemodynamic events in neurologically compromised infants: a simultaneous EEG and diffuse optical imaging study. Neuroimage (2011) 55:1610-6. doi: 10.1016/j.neuroimage.2011.01.022

62. Nemoto EM, Yonas H, Kassam A. Clinical experience with cerebral oximetry in stroke and cardiac arrest. Critical Care Med. (2000) 28:1052-4. doi: 10.1097/00003246-200004000-00023

63. Damian MS, Schlosser R. Bilateral near infrared spectroscopy in spaceoccupying middle cerebral artery stroke. Neurocritical Care (2007) 6:165-73. doi: 10.1007/s12028-007-0010-3

64. Sakatani K, Murata Y, Fujiwara N, Hoshino T, Nakamura S, Kano T, et al. Comparison of blood-oxygen-level-dependent functional magnetic resonance imaging and near-infrared spectroscopy recording during functional brain activation in patients with stroke and brain tumors. J Biomed Opt. (2007) 12:062110. doi: 10.1117/1.2823036

65. Aries MJ, Coumou AD, Elting JW, van der Harst JJ, Kremer BP, Vroomen PC. Near infrared spectroscopy for the detection of desaturations in vulnerable ischemic brain tissue: a pilot study at the stroke unit bedside. Stroke (2012) 43:1134-6. doi: 10.1161/STROKEAHA.111.636894

66. Mundiyanapurath S, Schonenberger S, Rosales ML, Carrilho Romeiro AM, Mohlenbruch M, Bendszus $M$, et al. Circulatory and respiratory parameters during acute endovascular stroke therapy in conscious sedation or general anesthesia. J Stroke Cerebrovasc Dis. (2015) 24:1244-9. doi: 10.1016/j.jstrokecerebrovasdis.2015.01.025

67. Liebig T, Gralla J, Schroth G. Endovascular treatment of acute stroke: evolution and selection of techniques and instruments based on thrombus imaging. Clin Neuroradiol. (2015) 25(Suppl. 2), 299-306. doi: 10.1007/s00062-015-0435-6

68. Hametner C, Stanarcevic P, Stampfl S, Rohde S, Veltkamp R, Bösel J. Noninvasive cerebral oximetry during endovascular therapy for acute ischemic stroke: an observational study. J Cereb Blood Flow Metab. (2015) 35:1722-8. doi: 10.1038/jcbfm.2015.181

69. Hashimoto T, Matsumoto S, Ando M, Chihara H, Tsujimoto A, Hatano T. Cerebral hyperperfusion syndrome after endovascular reperfusion therapy in a patient with acute internal carotid artery and middle cerebral artery occlusions. World Neurosurg. (2018) 110, 145-51. doi: 10.1016/j.wneu.2017.11.023

70. Ritzenthaler T, Cho TH, Luis D, Berthezene Y, Nighoghossian N. Usefulness of near-infrared spectroscopy in thrombectomy monitoring. J Clin Monit Comput. (2015) 29:585-9. doi: 10.1007/s10877-014-9636-9

71. Nakamura S, Sakatani K, Kano T, Hoshino T, Fujiwara N, Murata Y, et al. Effects of revascularisation on evoked cerebral blood oxygenation responses in stroke patients. Adv Exp Med Biol. (2010) 662, 525-30. doi: 10.1007/978-1-4419-1241-1_76

72. Vernieri F, Rosato N, Pauri F, Tibuzzi F, Passarelli F, Rossini PM. Near infrared spectroscopy and transcranial Doppler in monohemispheric stroke. Eur Neurol. (1999) 41:159-62. doi: 10.1159/000008041

73. Moreau F, Yang R, Nambiar V, Demchuk AM, Dunn JF. Near-infrared measurements of brain oxygenation in stroke. Neurophotonics (2016) 3:031403. doi: 10.1117/1.NPh.3.3.031403

74. Olsson C, Thelin S. Regional cerebral saturation monitoring with near-infrared spectroscopy during selective antegrade cerebral perfusion: diagnostic performance and relationship to postoperative stroke. J Thorac Cardiovasc Surg. (2006) 131:371-9. doi: 10.1016/j.jtcvs.2005.08.068

75. Nakamura S, Kano T, Sakatani K, Hoshino T, Fujiwara N, Murata Y, et al. Optical topography can predict occurrence of watershed infarction during carotid endarterectomy: technical case report. Surg Neurol. (2009) 71:540-2. doi: 10.1016/j.surneu.2007.11.012

76. Kellermann K, Jungwirth B. Avoiding stroke during cardiac surgery. Semin Cardiothorac Vasc Anesth. (2010) 14:95-101. doi: $10.1177 / 1089253210370902$

77. Pennekamp CW, Moll FL, de Borst GJ. The potential benefits and the role of cerebral monitoring in carotid endarterectomy. Curr Opin Anaesthesiol. (2011) 24:693-7. doi: 10.1097/ACO.0b013e32834c7aal

78. Ono M, Joshi B, Brady K, Easley RB, Zheng Y, Brown C, et al. Risks for impaired cerebral autoregulation during cardiopulmonary bypass and postoperative stroke. Br J Anaesth. (2012) 109:391-8. doi: 10.1093/bja/aes148

79. Rummel C, Zubler C, Schroth G, Gralla J, Hsieh K, Abela E, et al. Monitoring cerebral oxygenation during balloon occlusion with multichannel NIRS. J Cereb Blood Flow Metab. (2014) 34:347-56. doi: 10.1038/jcbfm.2013.207

80. Viola S, Viola P, Litterio P, Buongarzone MP, Fiorelli L. Stroke risk and migraine: near-infrared spectroscopy study. Neurol Sci. (2012) 33(Suppl. 1):S173-5. doi: 10.1007/s10072-012-1077-y

81. Li Z, Zhang M, Xin Q, Chen G, Liu F, Li J. Spectral analysis of near-infrared spectroscopy signals measured from prefrontal lobe in subjects at risk for stroke. Med Phys. (2012) 39:2179-85. doi: 10.1118/1.3696363

82. Pizza F, Biallas M, Kallweit U, Wolf M, Bassetti CL. Cerebral hemodynamic changes in stroke during sleep-disordered breathing. Stroke (2012) 43:19513. doi: 10.1161/STROKEAHA. 112.656298

83. Selb J, Yucel MA, Phillip D, Schytz HW, Iversen HK, Vangel M, et al. Effect of motion artifacts and their correction on near-infrared spectroscopy oscillation data: a study in healthy subjects and stroke patients. J Biomed Opt. (2015) 20:56011. doi: 10.1117/1.JBO.20.5.056011

84. Chaudhary U, Birbaumer N, Ramos-Murguialday A. Brain-computer interfaces in the completely locked-in state and chronic stroke. Progr Brain Res. (2016) 228:131-61. doi: 10.1016/bs.pbr.2016.04.019

85. Coyle S, Ward, T, Markham C. On the suitability of near-infrared (NIR) systems for next-generation brain-computer interfaces. Physiol Measure. (2004) 25:815-822. doi: 10.1088/0967-3334/25/4/003

86. Coyle SM, Ward TE, Markham CM. Brain-computer interface using a simplified functional near-infrared spectroscopy system. J Neur Eng. (2007) 4:219-26. doi: 10.1088/1741-2560/4/3/007

87. Sitaram R, Zhang H, Guan C, Thulasidas M, Hoshi Y, Ishikawa A, et al. Temporal classification of multichannel near-infrared spectroscopy signals of motor imagery for developing a brain-computer interface. Neuroimage (2007) 34:1416-27. doi: 10.1016/j.neuroimage.2006.11.005

88. Lisa H, Martin W. Single-trial classification of motor imagery differing in task complexity: a functional near-infrared spectroscopy study. J NeuroEng Rehabil. (2011) 8:34-34. doi: 10.1186/1743-0003-8-34

89. Naseer N, Hong KS. Classification of functional near-infrared spectroscopy signals corresponding to the right- and left-wrist motor imagery for development of a brain-computer interface. Neurosci Lett. (2013) 553:84-9. doi: 10.1016/j.neulet.2013.08.021 
90. Hong KS, Naseer N, Kim YH. Classification of prefrontal and motor cortex signals for three-class fNIRS-BCI. Neurosci Lett. (2015) 587:87-92. doi: 10.1016/j.neulet.2014.12.029

91. Fazli S, Mehnert J, Steinbrink J, Curio G, Villringer A, Müller KR, et al. Enhanced performance by a hybrid NIRS-EEG brain computer interface. Neuroimage (2012) 59:519-29. doi: 10.1016/j.neuroimage.2011. 07.084

92. Khan MJ, Hong KS. Hybrid EEG-fNIRS-based eight-command decoding for BCI: application to quadcopter control. Front Neurorobot. (2017) 11:6. doi: 10.3389/fnbot.2017.00006

93. Hong KS, Khan MJ. Hybrid brain-computer interface techniques for improved classification accuracy and increased number of commands: a review. Front Neurorobot. (2017) 11:35. doi: 10.3389/fnbot.2017.00035

94. Hong KS, Khan MJ, Hong MJ. Feature extraction and classification methods for hybrid fNIRS-EEG brain-computer interfaces. Front Hum Neurosci. (2018) 12:246. doi: 10.3389/fnhum.2018.00246

95. Koo B, Lee HG, Nam Y, Kang H, Koh CS, Shin HC, et al. A hybrid NIRS-EEG system for self-paced brain computer interface with online motor imagery. J Neurosci Methods (2015) 244(Sp. Iss. SI):26-32. doi: 10.1016/j.jneumeth.2014.04.016

96. Yin X, Xu B, Jiang C, Fu Y, Wang Z, Li H, et al. A hybrid BCI based on EEG and ANIRS signals improves the performance of decoding motor imagery of both force and speed of hand clenching. J Neural Eng. (2015) 12:036004. doi: 10.1088/1741-2560/12/3/036004

97. Buccino AP, Keles HO, Omurtag A. Plos ONE (2016) doi: 10.1371/journal.pone.0146610.11:e0146610.

98. Kirilina E, Jelzow A, Heine A, Niessing M, Wabnitz H, Brühl R, et al. The physiological origin of task-evoked systemic artefacts in functional near infrared spectroscopy. Neuroimage (2012) 61:70-81. doi: 10.1016/j.neuroimage.2012.02.074

99. Caldwell M, Scholkmann F, Wolf U, Wolf M, Elwell C, Tachtsidis I. Modelling confounding effects from extracerebral contamination and systemic factors on functional near-infrared spectroscopy. NeuroImage (2016) 143:91-105. doi: 10.1016/j.neuroimage. 2016.08.05

100. Zimeo Morais GA, Scholkmann F, Balardin JB, Furucho RA, de Paula RCV, Biazoli CE, et al. Non-neuronal evoked and spontaneous hemodynamic changes in the anterior temporal region of the human head may lead to misinterpretations of functional near-infrared spectroscopy signals. Neurophotonics (2018) 5:011002. doi: 10.1117/1.NPh.5.1.011002

101. Nguyen HD, Hong KS. Bundled-optode implementation for 3D imaging in functional near-infrared spectroscopy. Biomedical Optics Express (2016) 7:3491. doi: 10.1364/BOE.7.003491

102. Nguyen HD, Hong KS, Shin YI. Bundled-optode method in functional near-infrared spectroscopy. PLoS ONE (2016) 11:e0165146. doi: 10.1371/journal.pone.0165146

103. Zafar A, Hong KS. Detection and classification of three-class initial dips from prefrontal cortex. Biomedical Optics Express (2016) 8:367. doi: 10.1364/BOE.8.000367
104. Zafar A, Hong KS. Neuronal activation detection using vector phase analysis with dual threshold circles: a functional near-infrared spectroscopy study. Neural Syst. (2018) 24:1850031. doi: 10.1142/S0129065718500314

105. Gallerani M, Manfredini R, Ricci L, Cocurullo A, Goldoni C, Bigoni M, et al. Chronobiological aspects of acute cerebrovascular diseases. Acta Neurologica Scand. (2010) 87:482-7. doi: 10.1111/j.1600-0404.1993.tb04141.x

106. Calderon-Arnulphi M, Alaraj A, Amin-Hanjani S, Mantulin WW, Polzonetti $\mathrm{CM}$, Gratton E, et al. Detection of cerebral ischemia in neurovascular surgery using quantitative frequency-domain near-infrared spectroscopy. $J$ Neurosurg. (2007) 106:283-90. doi: 10.3171/jns.2007.106.2.283

107. Eliassen JC, Boespflug EL, Lamy M, Allendorfer J, Chu WJ, Szaflarski JP. Brain-mapping techniques for evaluating poststroke recovery and rehabilitation: a review. Topics Stroke Rehabil. (2008) 15:427-50. doi: 10.1310/tsr1505-427

108. Nitsche MA, Paulus W. Excitability changes induced in the human motor cortex by weak transcranial direct current stimulation. J Physiol. (2000) 527:633-9.

109. Roy A, Baxter B, He B. High-definition transcranial direct current stimulation induces both acute and persistent changes in broadband cortical synchronization: A simultaneous tDCS-EEG study. IEEE Trans. Biomed. Eng. (2014). 61:1967-78. doi: 10.1109/TBME.2014.2311071

110. Kasashima Y, Fujiwara T, Matsushika Y, Tsuji T, Hase K, Ushiyama $\mathrm{J}$, et al. Modulation of event-related desynchronization during motor imagery with transcranial direct current stimulation (tDCS) in patients with chronic hemiparetic stroke. Exp Brain Res. (2012) 221:263-8. doi: 10.1007/s00221-012-3166-9

111. Rahman A, Reato D, Arlotti M, Gasca F, Datta A, Parra LC, et al. Cellular effects of acute direct current stimulation: somatic and synaptic terminal effects. J Physiol (Lond) (2013) 591(Pt 10):2563-78. doi: $10.1113 /$ jphysiol.2012.247171

112. Jones KT, Gözenman F, Berryhill ME. The strategy and motivational influences on the beneficial effect of neurostimulation: a tDCS and fNIRS study. Neuroimage (2015) 105:238-47. doi: 10.1016/j.neuroimage.2014.11.012

113. Yaqub MA, Woo SW, Hong KS. Effects of HD-tDCS on resting-state functional connectivity in the prefrontal cortex: an fNIRS study. Complexity (2018) 2018:1613402. doi: 10.1155/2018/1613402

Conflict of Interest Statement: The authors declare that the research was conducted in the absence of any commercial or financial relationships that could be construed as a potential conflict of interest.

Copyright (C) 2019 Yang, Yang, Yuan, Feng and Wang. This is an open-access article distributed under the terms of the Creative Commons Attribution License (CC BY). The use, distribution or reproduction in other forums is permitted, provided the original author(s) and the copyright owner(s) are credited and that the original publication in this journal is cited, in accordance with accepted academic practice. No use, distribution or reproduction is permitted which does not comply with these terms. 